Please cite this article as:

A. Romero, J. González, R. Picos, M. J. Deen, J. A. Jiménez-Tejada, Evolutionary parameter extraction for an organic TFT compact model including contact effects, Organic Electronics, (2018), 61, 242-253.

(C) 2018. This manuscript version is made available under the CC-BY-NC-ND 4.0 license http://creativecommons.org/licenses/by-nc-nd/4.0/

Digital Object Identifier:

10.1016/j.orgel.2018.05.062

Source:

https://www.sciencedirect.com/science/article/pii/S1566119918302908 


\title{
Evolutionary Parameter Extraction for an Organic TFT Compact Model including Contact Effects $^{\text {is }}$
}

\author{
A. Romero ${ }^{\mathrm{a}, \mathrm{d}}$, J. González ${ }^{\mathrm{a}}$, R. Picos ${ }^{\mathrm{b}}$, M. J. Deen ${ }^{\mathrm{c}}$, J. A. Jiménez-Tejada ${ }^{\mathrm{d}, *}$ \\ ${ }^{a}$ Departamento de Arquitectura y Tecnología de Computadores, CITIC-UGR, Universidad de Granada, Granada 18071, Spain \\ ${ }^{b}$ GTE, University of Balearic Islands, Palma 07122, Spain \\ ${ }^{c}$ Department of Electrical and Computer Engineering, McMaster University, Hamilton, ON L8S 4K1 Canada \\ ${ }^{d}$ Departamento de Electrónica y Tecnología de los Computadores, CITIC-UGR, Universidad de Granada, Granada 18071, Spain
}

\begin{abstract}
In this work, an evolutionary parameter extraction procedure is combined with a compact model for the current-voltage characteristics of organic thin-film transistors as a part of a circuit design tool to extract the device parameters. This procedure can be used to overcome shortcomings of previous parameter extraction procedures. The proposed evolutionary procedure can be used in those situations whereby the parameter set extracted by other procedures does not comply its physical meaning, or if a poor agreement between the experimental data and the analytical results exists. In the last case, the evolutionary procedure can be used as a problem optimization method. After the evolutionary parameter extraction procedure is applied to the transistor output characteristics, the obtained results show an excellent agreement with the experimental data.
\end{abstract}

Keywords: OTFT, device parameters, contact effects, evolutionary computation

\section{Introduction}

Organic thin-film transistors (OTFTs) have attracted considerable research interest because of the advantages associated to the materials used, such as flexibility, low fabrication costs and weight, and their potential applications in large-area, flexible electronics, such as displays and sensors [1]. Nevertheless, they also have important limitations, for example a low carrier mobility compared to inorganic TFTs ([1-3]), high process variability $[4,5]$, or degraded performance characteristics due to contact effects. Contact effects, specifically, have been extensively studied in order to introduce them in transistor compact models [6-14].

Compact models are analytical models that are able to reproduce the electrical behavior of a given device in all regions of operation. They are usually based on physical principles, with some free parameters that are used to stitch the transition between regions, or to improve the needed mathematical simplification of the underlying device physics. Thus, these models must be associated with methods to extract their respective parameters from the electrical characteristics of a transistor $[6,7,9,11,15]$. Parameter extraction procedures must assign a correct physical value to each parameter in order to keep

\footnotetext{
This work was supported by projects TIN2015-67020-P, MAT201676892-C3-3-R, TEC2014-56244-R, and Excellence Networks REFLEXIO and NANOVAR funded by the Spanish "Ministerio de Economía y Competitividad" and European Regional Development Funds (ERDF).

* Corresponding author

Email addresses: ad90ry@correo.ugr .es (A. Romero), jesusgonzalez@ugr .es (J. González), rodrigo.picos@uib.es (R. Picos), jamal@mcmaster.ca (M. J. Deen), tejada@ugr.es (J. A. Jiménez-Tejada)
}

their meaning. An invalid value would cause the parameter extraction procedure to fail or the achievement of an unphysical parameter set. Finally, the extracted parameter set has to provide a good agreement between the model and the experimental data. Typically, this parameter extraction is usually a two-step procedure, because the parameters are usually correlated. In a first step, a direct extraction method under some simplifying assumptions is used to obtain a first set of parameters. In a second step, this set may be used in a global optimization procedure.

In this work, we consider a compact model [11], which has been successfully tested in the past with different OTFTs operating in different regions [16-18] and even in transistors showing current-voltage curves with hysteresis [19]. The application of this model can also be found in two-dimensional field effect transistors, in which the contact effects clearly affect the device performance $[20,21]$. A procedure that accelerates the extraction of the parameters of this model, or solves some of the problems noted in the past [22], would be very helpful. For this reason, in this paper an evolutionary parameter extraction procedure is proposed, solving the extraction shortcomings or weaknesses of the procedure proposed in [11]. Our procedure is based on a special kind of heuristic and optimization search technique called Evolutionary Algorithms (EAs). EAs are employed in a broad range of engineering disciplines [23] such as architecture [24, 25], electrical [26, 27], chemical [28, 29] and mechanical engineering [30,31]. EAs are also employed in other important fields as computer science [32], control [33] and signal processing [34]. Moreover, EAs have been previously used to extract the parameters of semiconductor devices, such as MOSFETs [27, 35-38], photovoltaic (PV) modules $[39,40]$, or solar cells $[31,39,41]$. They have even been used 
to extract parameters for simple OTFTs models [42-44]. The widespread use of EAs in different fields along with its capacity to be employed as a parameter extraction procedure, makes EAs an interesting option to be used for the procedure presented in [11] to gain in accuracy and speed.

For these reasons, we propose to use EAs in combination with the procedure introduced in [11]. This paper is organized as follows. In Section 2, the features and possible limitations of the direct parameter extraction procedure proposed in [11], when applied to experimental data, is shown. Since an evolutionary procedure is going to be presented in future sections, a brief review of EAs is given in Section 3. In Section 4, an introduction of Multi-objective Problems (MOPs) is presented. Then, in Section 5, the combination of concepts presented in Sections 3 and 4 is reviewed. In Section 6, the evolutionary procedure is developed for the compact model [11]. In Section 7, the evolutionary procedure is tested with experimental data. Finally, the conclusions are presented in Section 8.

\section{Parameter Extraction Procedure}

In $[45,46]$, a compact model was developed for OTFTs which includes the voltage drop at the source contact $\left(V_{S} \equiv V_{C}\right)$ and electric field dependent mobility $\mu=\mu_{0}\left(V_{G}-V_{T}\right)^{\gamma}$ :

$$
\begin{aligned}
I_{D} & =-k^{\prime} \frac{W}{L} \frac{\left[\left(V_{G}-V_{T}-V_{S}\right)^{\gamma+2}-\left(V_{G}-V_{T}-V_{D}\right)^{\gamma+2}\right]}{\gamma+2} \\
k^{\prime} & =\mu_{0} C_{o x}
\end{aligned}
$$

where $V_{G}$ is the gate voltage, $V_{D}$ is the drain voltage, $V_{T}$ is the threshold voltage, $\gamma$ is the mobility enhancement factor, $\mu_{0}$ is the mobility-related parameter and its dimension is expressed as $\mathrm{cm}^{2} /\left(\mathrm{V}^{1+\gamma} \mathrm{s}\right), C_{o x}$ is the capacitance per unit area of the oxide and $W$ and $L$ are the channel width and length, respectively.

Later on, model (1) was redefined with the inclusion of a model for the contact region [11]:

$$
V_{S}=\left(\frac{I_{D}}{M_{C}}\right)^{\frac{1}{m_{k}}}
$$

where the parameter $M_{C}$ is usually gate voltage dependent and $m_{k}$ indicates the grade of the observed trend (from linear to quadratic, $1 \leq m_{k} \leq 2$ ) in the triode region of the OTFT. In the case of a linear trend $\left(m_{k}=1\right), M_{C}$ would be equivalent to the inverse of a contact resistance $R_{C}$. When this occurs, the $I_{D}-V_{S}$ relation can be approximated by the usual linear relation

$$
V_{S}=I_{D} R_{C}
$$

The more general expression (2) for the $I_{D}-V_{S}$ relation was proposed in order to include linear and non-linear relations as observed experimentally in OTFTs [47] and after theoretical studies on metal-organic contacts in which the transition from linear to quadratic trends was analyzed [9, 48].

Along with (1) and (2), the authors proposed a method to extract the parameters of (1) and the voltage drop at the contact $I_{D}-V_{S}$ from experimental $I_{D}-V_{D}$ curves [11]. The method makes use of the so-called $H_{V G}$ function [45, 46], with the objective of getting an initial estimation of the values of $\gamma$ and $V_{T}$. It is defined as the ratio of the integral of the drain current $\left(I_{D}\right)$ over the gate bias divided by the drain current

$$
H_{V G}\left(V_{G}\right)=\frac{\int_{<V_{T}}^{V_{G}} I_{D}\left(V_{G}\right) d V_{G}}{I_{D}\left(V_{G}\right)}
$$

The $H_{V G}$ function can be evaluated from experimental data measured in the linear and saturation regimes. Combining (1) and (4), a linear function with $V_{G}$ is obtained for the saturation regime [46]

$$
H_{V G}\left(V_{G}\right)=\left(V_{G}-V_{T}-V_{S}\right) /(\gamma+3)
$$

The linear expression (5) can be used to get an initial estimation of the values of $\gamma$ and $V_{T}$ incorporating the experimental values of $H_{V G}$ and assuming $V_{S}$ as a constant value, usually $V_{S}=0$. However, $V_{S}$ is a function of $I_{D}$ and $V_{G}\left(V_{S}=V_{S}\left(I_{D}, V_{G}\right)\right)$. Thus, the values of $\gamma$ and $V_{T}$ incorporate any inaccuracy coming from the assumed value of $V_{S}$. After this initial estimation of $\gamma$ and $V_{T}$, if $k^{\prime}$ is also known, the voltage in the contacts $V_{S}\left(I_{D}, V_{G}\right)$ can be extracted by introducing the values of the experimental data and the values of $\gamma, V_{T}$ and $k^{\prime}$ in (1):

$$
\begin{aligned}
V_{S}= & V_{G}-V_{T} \\
& -\left[I_{D}(\gamma+2) L /\left(W k^{\prime}\right)+\left(V_{G}-V_{T}-V_{D}\right)^{\gamma+2}\right]^{1 /(\gamma+2)}
\end{aligned}
$$

Since the $k^{\prime}$ value is unknown, various values are tested and introduced in (6) to find the relation $I_{D}-V_{S}$. The initial range of values for $k^{\prime}$ can vary by two orders of magnitude over and below the value obtained with the ideal MOS model applied to the experimental $I_{D}-V_{G}$ data in the saturation regime. The obtained relation $I_{D}-V_{S}$ must have physical meaning according to (2). Only the values of $k^{\prime}$ that make the relation $I_{D}-V_{S}$ follow (2) are considered. An averaged value of the contact voltage obtained from this relation $I_{D}-V_{S}$ must be consistent with the initially assumed value of $V_{S}$ in the above paragraph. Otherwise, this new averaged value of $V_{S}$ must be inserted in (5) and the process must be repeated until these conditions are fulfilled.

Although the compact model along with the parameter extraction procedure have been successfully used in the past $[11,16-19]$, it has been noticed that the $H_{V G}$ function may not always perform under certain circumstances:

- The values of $\gamma$ and $V_{T}$ extracted from (5) are inaccurate if $V_{C} \neq 0$.

- As initially defined in [49], the integral (4) must necessarily be evaluated from a gate voltage under the threshold voltage. Otherwise, the extracted values of $\gamma$ and $V_{T}$ will differ from the actual ones.

- The integral (4) performed with very few points (very few output characteristic curves) also leads to inaccurate values of $\gamma$ and $V_{T}$. 


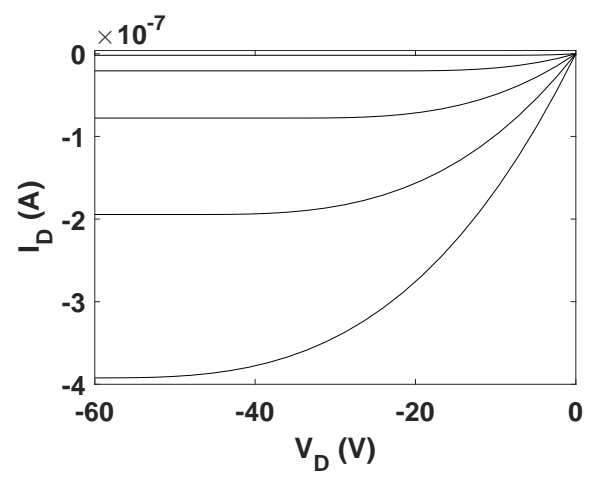

Figure 1: Output characteristics generated using the compact model (1) and the relation (2) at gate voltages $V_{G}$ from 0 to $-50 \mathrm{~V}$ with a $-12.5 \mathrm{~V}$ step (from top to bottom). $V_{D}$ is swept from 0 to $-60 \mathrm{~V}$ with a $-2.4 \mathrm{~V}$ step. $k^{\prime}=1.381 \times 10^{-14} \mathrm{~A} / \mathrm{V}^{2+\gamma}, W=2 \times 10^{-1} \mathrm{~cm}, L=5.0 \times 10^{-4} \mathrm{~cm}, V_{T}=10 \mathrm{~V}$, $\gamma=1, M_{C}\left(V_{G}\right)=\alpha\left|V_{G}-V_{T}\right|^{(\gamma+1)} \mathrm{V}^{m_{k}}$ with $\alpha=5 \times 10^{-10}$, and $m_{k}=1.2$. The contact voltage $V_{S}$ in the saturation region at $V_{D}=-60 \mathrm{~V}$ for each curve is $-0.06,-0.12,-0.17,-0.23,-0.28 \mathrm{~V}$ (from top to bottom).

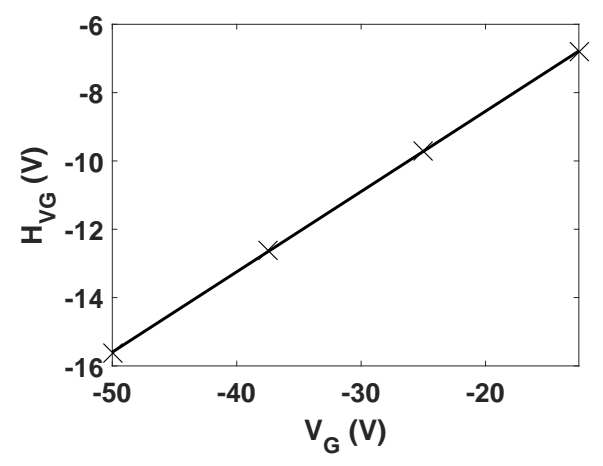

Figure 2: $H_{V G}$ function evaluated from the $I_{D}-V_{G}$ relation extracted at $V_{D}=$ $-60 \mathrm{~V}$ from the output characteristics of Fig. 1 (symbols). The solid line is the fitting with (5) using $V_{T}=16.35 \mathrm{~V}$ and $\gamma=1.25$.

In these cases, the errors in the values of $V_{T}$ and $\gamma$ can propagate through the next steps of the extraction procedure, providing a non-optimized parameter set $\left(k^{\prime}, \gamma, V_{T}, m_{k}, M_{C}\right)$.

An example of misuse of the $H_{V G}$ function can be seen in the analysis of the output characteristics $I_{D}-V_{D}$ of Fig. 1 . These curves have been generated using the compact model (1), the relation (2) and the bisection method [50]. Once the experimental data is available, $H_{V G}$ is applied to the saturation region $\left(V_{D}=-60 \mathrm{~V}\right)$. The result is represented with crosses in Fig. 2. After fitting the experimental $H_{V G}$ function with (5), $V_{T}=16.35 \mathrm{~V}$ and $\gamma=1.25$ are obtained. These values do not correspond with the $V_{T}$ and $\gamma$ values initially employed in Fig. 1, even for this case in which the contact voltage is much smaller than the drain voltage (values of the contact voltage are provided in Fig. 1). At this point, the parameter extraction procedure should be aborted since the following steps depend on the values extracted from the $H_{V G}$ function.

It is clear that solutions to solve this problem at the experimental laboratory exist: it is easy to measure transfer curves or more output characteristics, and that some of these curves

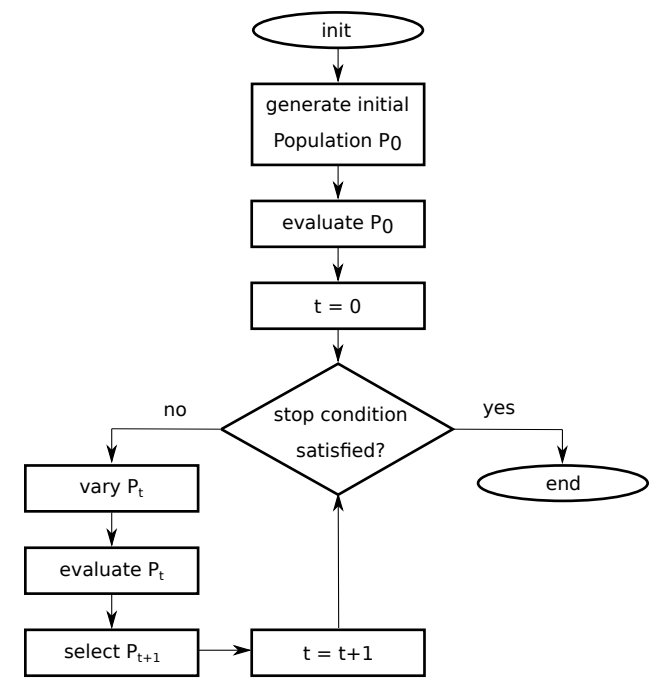

Figure 3: Basic flow chart of an EA.

may correspond to gate voltages below the threshold voltage. However, there may be situations in which these solutions are no longer possible or difficult to accomplish. In these cases, we propose to adapt the procedure in [11]. As alternative, an evolutionary parameter extraction procedure is proposed in Section 6. Before the evolutionary procedure is introduced, a general idea about the operation of EAs and their main mechanisms are presented in the next section.

\section{Evolutionary Algorithms}

As stated in the introduction, once a set of parameters has been determined by a direct method ([45, 46, 49, 51-53]), the parameter extraction can be improved by a global optimization process that takes into account possible interactions between the parameters. Different techniques can be used for this global optimization, which are usually hampered by the high dimensionality of the problem (the set of parameters to be extracted or optimized are usually composed of tens of elements). Initially, this was dealt with using deterministic methods, based on derivatives and local optimization $[54,55]$. Soon after, more efficient techniques based on evolutionary algorithms were proposed ([27, 35-38, 56]). Alternative methods, although less used than evolutionary computing, have also been proposed, such as a procedure that introduces expert knowledge into the parameter extraction for an OTFT compact model [15], or the particle-swarm-optimization method employed to extract the model parameters of the PSP compact MOSFET model [57].

EAs are heuristic search and optimization techniques based on natural and genetic evolution $[58,59]$. The concept of natural evolution will help the reader to get an initial idea about how EAs work. In nature, there are entities or populations of individuals with the ability of reproduction. These individuals usually have different characteristics, such as height or strength. The offspring individuals inherit some characteristics of their progenitors. In addition, some of them may mutate acquiring new 
qualities. As time passes, only the individuals able to adapt to the environment survive, usually the fittest ones. Taking as reference the natural evolution, EAs process at each iteration a population of potential solutions (individuals) for a determined problem (environment). During the EA execution the potential solutions are combined (reproduction) and some of them may be altered (mutation), giving rise to an offspring population, in which the fittest potential solutions have the highest probability of remaining in it (adaptation to the environment).

The most important difference between classical optimization techniques and EAs is that they process a population of potential solutions at each iteration rather than a single solution for the problem. Fig. 3 shows the basic flow chart of an EA, where $P_{t}$ is the population of the EA in the $t$-th iteration and $N$ is the population size. The individuals of the population are potential solutions of a minimization (maximization) function $f(x)$. They are named $x^{(i, t)}=\left(x_{1}{ }^{(i, t)}, \ldots, x_{p}{ }^{(i, t)}\right), i=1,2, \ldots, N$ and $x^{(i, t)} \in X^{p} \subseteq \mathbb{R}^{p}$, where $X^{p}$ is the domain of the population.

Three main operators guide the evolution of the population:

- Recombination operator. It is mainly responsible for the population to improve. This operator mixes characteristics of progenitors in order to create new individuals, called offsprings. The idea of recombination is to discover better individuals than the original ones, or intermediate individuals that in future would allow to discover better ones.

- Mutation operator. This operator contributes to the diversity of the population. The recombination of progenitors by itself may not improve the population or may not discover new individuals. In these cases, some of the offsprings should be altered by other means. The mutation operator is responsible for securing that every individual from the search space is achievable. This operator is also used by the EA to evade from local optimum solutions.

- Selection operator. Based on a measure of adequacy of the individuals, this operator decides which individuals will be part of the next generation population.

EAs are highly parameterized algorithms, being their most important features introduced in the next subsections.

\subsection{Individual Representation}

The first step in defining an EA is to link the original problem context and the problem solving space where evolution takes place. Very importantly, it has to be clear what kind of solutions the original problem has. Then, the most appropriate domain for them is defined, and finally the most appropriate individual representation for it is used.

\subsection{Initial Population}

At the beginning of an EA, the population is empty. In order to fill it with individuals, a method to generate the initial population $P_{0}$ has to be defined. There is no standard method to initialize the population of an EA and usually $P_{0}$ is randomly generated. Also, an old population or a population from another heuristic could be used as $P_{0}$.

\subsection{Variation Operators}

The recombination and mutation operators are defined with the recombination $\left(P_{c}\right)$ and mutation $\left(P_{m}\right)$ rates, respectively. Through the use of the right values of $P_{c}$ and $P_{m}$, the complete search space should be explored. A low $P_{c}$ will not allow to explore the complete search space, while a high $P_{m}$ will avoid the convergence of the EA.

\subsection{Fitness Function}

The fitness function indicates the differences in quality among the individuals of the population. It gives a punctuation to each individual of the population. Given a minimization (or maximization) function, an individual $x^{q}$ will get a lower (or greater) punctuation than other $x^{j}$ if $x^{q}$ is a better individual.

\subsection{Selection Operator}

The selection operator selects the fittest or the best punctuated individuals, which will be part of the population of the next generation $P_{t+1}$. Moreover, $P_{t+1}$ must meet certain requirements, such as diversity and elitism.

\subsubsection{Diversity}

The diversity of the population is a very important fact in complex optimization problems such as the one presented in this work. If all individuals in the population were located in the same space, for example if $P_{t+1}$ were composed only of the fittest individuals, the procedure could converge prematurely to local optima. On the other hand, if $P_{t+1}$ were composed of bad individuals, it would not converge at all. The selection operator controls the commitment between quality and diversity among individuals in the population in order to ensure a proper convergence. The fittest individuals are given a higher probability to take part in $P_{t+1}$ than the worst individuals. Nevertheless, bad individuals also have a chance of being in $P_{t+1}$.

\subsubsection{Elitism}

Since EAs are stochastic, the best individual found so far, $x^{\text {best }}$, may be lost during the evolving procedure. EAs introduce elitism to guarantee the presence of $x^{\text {best }}$ in $P_{t+1}$. During the evolving procedure and at the end of the execution, $x^{\text {best }}$ is always available.

\subsection{Stop Condition}

Another critical parameter in the EA definition is to decide when to stop it. For that purpose, one the following criteria should be fulfilled:

- Global optimum solution achieved. In this case, it makes no sense to continue searching for a better solution.

- $C P U$ cost. In order to minimize the computational time, the EA execution can be restricted to a limited number of generations and evaluations of the fitness function.

- Stagnation of the search. The EA execution can be stopped after a limited number of generations if there is no improvement in the population. In this case, the population should be restarted, but keeping $x^{\text {best }}$. 


\section{Multi-objective Problems}

In most practical situations, problems have various requirements to accomplish. Parameter extraction procedure is only one of them. Problems of this kind are known as multi-objective problems (MOPs) [60]. The greatest difference between single objective problems and MOPs is the arrangement of the set of potential solutions. In a problem with $n_{o b j}$ competing objectives, each one of them is measured by an objective function $f_{i}\left(i=1, \ldots, n_{o b j}\right)$. We can define a global objective function $f$ that meets the following relations for two potential solutions for the problem $s_{1}$ and $s_{2}$

$$
\begin{aligned}
& f\left(s_{1}\right)=f\left(s_{2}\right) \Leftrightarrow f_{i}\left(s_{1}\right)=f_{i}\left(s_{2}\right) \forall i \in 1,2, \ldots, n_{o b j} \\
& f\left(s_{1}\right) \leq f\left(s_{2}\right) \Leftrightarrow f_{i}\left(s_{1}\right) \leq f_{i}\left(s_{2}\right) \forall i \in 1,2, \ldots, n_{o b j} \\
& f\left(s_{1}\right)<f\left(s_{2}\right) \Leftrightarrow f\left(s_{1}\right) \leq f\left(s_{2}\right) \wedge f\left(s_{1}\right) \neq f\left(s_{2}\right)
\end{aligned}
$$

Taking into account the above relations, the Paretodominance criterion can be used to establish an order between the individuals of the population [61]:

$$
\begin{aligned}
& s_{1} \prec s_{2} \Leftrightarrow f\left(s_{1}\right)<f\left(s_{2}\right) \\
& s_{1} \leq s_{2} \Leftrightarrow f\left(s_{1}\right) \leq f\left(s_{2}\right) \\
& s_{1} \sim s_{2} \Leftrightarrow f\left(s_{1}\right) \not \leq f\left(s_{2}\right) \wedge f\left(s_{2}\right) \not \leq f\left(s_{1}\right)
\end{aligned}
$$

where $s_{1} \prec s_{2}$ ( $s_{1}$ dominates $\left.s_{2}\right)$ means that $s_{1}$ is a better solution than $s_{2}, s_{1} \leq s_{2}\left(s_{1}\right.$ weakly dominates $\left.s_{2}\right)$ means that $s_{1}$ is a better or equal solution than $s_{2}$, and $s_{1} \sim s_{2}$ ( $s_{1}$ is indifferent to $s_{2}$ ) means that both solutions are not comparable. A Paretooptimum solution $s_{\text {opt }}[61]$ is defined as a solution which cannot be dominated by any other solution in the solution set $S$ :

$$
\nexists s_{i} \in S: s_{i}<s_{\text {opt }}
$$

All Pareto-optimum solutions compose the Pareto front. The solutions of the Pareto front are indifferent to each other and equally valid. Thus, the criteria of the final users or experts will decide which solutions from the Pareto front are the best.

\section{Multi-objective Evolutionary Algorithms}

The combined use of EAs and the Pareto-dominance criterion gives rise to a special type of EAs, called Multiobjective Evolutionary Algorithms (MOEAs), capable of managing MOPs. MOEAs follow the same principles of EAs (described in Fig. 3) but including the mechanisms needed to operate with MOPs.

There are many ways of implementing a MOEA [6265]. The Non-dominated Sorting Genetic Algorithm II (NSGA-II) [62] has been used successfully in a wide variety of problems and also employed in this work. The main objective of NSGA-II is to find a generation $P_{t+1}$ of $N$ individuals from a previous population $P_{t}$ of $N$ individuals also. The evolution between generations is developed with the selection, recombination and mutation operators and other criteria defined below.
- Non-Dominated sort. The population is sorted with the non-domination criterion. The individuals of the population are grouped in different fronts $F_{i}(i=0,1, \ldots)$, where $i$ indicates the non-domination level, $F_{0}$ is the front of individuals dominating the rest of solutions, $F_{1}$ is the next front, only dominated by $F_{0}$, and so on.

- Crowding distance. It measures how close to its neighbors an individual is. In order to reduce the agglomeration of individuals, a large crowding distance is necessary. The control of this distance is used to improve the diversity of the population.

- Selection operator. NSGA-II uses the tournament selection operator [66]. This operator chooses randomly $k$ individuals from $P_{t}$, and from these $k$ individuals the one situated in the best front is selected. In the case that the $k$ individuals are located in the same front, the one with the highest crowding distance is selected. This process is repeated until a population $Q_{t}$ of $N$ individuals is available. The population $Q_{t}$ is transformed with the recombination and mutation operators, which are defined in the next section. Finally a population $R_{t}$ is built by joining $P_{t}$ and $Q_{t}$, $R_{t}=P_{t} \cup Q_{t}$.

- Elitism and population of the next iteration. The population of the next generation $P_{t+1}$ is formed with the individuals of the best fronts of $R_{t}$ (truncating the number of individuals to $N$ ).

\section{Evolutionary Parameter Extraction Procedure}

As described in previous sections, the aim of this work is to design an evolutionary procedure capable of extracting the parameters of an OTFT from experimental output characteristics using the model (1)-(2). Now, the parameters needed to adapt NSGA-II to this objective are detailed.

\subsection{Individual Representation}

Our procedure is based on a set of experimental data $I_{D}=I_{D}\left(V_{G_{i}}, V_{D_{j}}\right)$, where $i \in \mathbb{Z}: 1 \leq i \leq g$ and $j \in \mathbb{Z}: 1 \leq j \leq d$. The numerical estimation of $I_{D}$ and of the voltage drop at the contact region with (1)-(2) are named $\widehat{I_{D}}\left(V_{G_{i}}, V_{D_{j}}, x\right)$ and $\widehat{V_{S}}\left(V_{G_{i}}, V_{D_{j}}, x\right)$, respectively, where $x$ refers to the rest of parameters needed to compute (1)-(2) and is defined as the individual of the population:

$$
x=\left(k^{\prime}, \gamma, V_{T}, \widehat{M_{C}}\left(V_{G_{1}}\right), \ldots, \widehat{M_{C}}\left(V_{G_{g}}\right)\right)
$$

where $g$ is the number of different $V_{G}$ values employed to generate $I_{D}, \widehat{M_{C}}\left(V_{G_{i}}\right)$ is the estimation of $M_{C}\left(V_{G}\right)$ at these particular values of $V_{G}$ and the length or number of variables of the individual (10) is $p=g+3$.

The parameter $m_{k}$ controls the linearity of the $I_{D}-V_{S}$ curves and does not depend on the gate voltage [11, 19]. A simple inspection of the output characteristics in the triode region can provide a first estimation of $m_{k}$. Intentionally, the parameter $m_{k}$ does not form part of the individual (10), but will be a static 
predefined parameter of the evolutionary procedure. The reason is that the joint use of $m_{k}$ and the $\widehat{M_{C}}\left(V_{G}\right)$ in (10) could cause the no-convergence to an acceptable solution.

\subsection{Recombination Operator}

The SBX operator is employed in the recombination operation for its successful performance at continuous search spaces [67, 68]. All individuals of the population $Q_{t}$, previously selected from $P_{t}$, participate in the recombination process, but only half $\left(P_{c}=0.5\right)$ of the variables of an individual will be recombined. This operator takes randomly from the population $N / 2$ pairs of parent individuals $x^{j}$ and $x^{q}$, which give place to two offspring individuals $c^{j}$ and $c^{q}$. Each variable $i$ of both parents is recombined if a random number $u$ taken within $[0,1]$ is greater than $P_{c}$. In the opposite case, the variable $i$ of each offspring individual will be a copy of the variable $i$ of a respective progenitor. In the first case, each offspring variable is symmetrically calculated as

$$
\begin{aligned}
c_{i}^{j} & =\bar{x}_{i}-\frac{1}{2} \beta\left(x_{i}^{q}-x_{i}^{j}\right), \\
c_{i}^{q} & =\bar{x}_{i}+\frac{1}{2} \beta\left(x_{i}^{q}-x_{i}^{j}\right)
\end{aligned}
$$

where $\bar{x}_{i}=\left(x_{i}^{j}+x_{i}^{p}\right) / 2$ and $\beta$ is a polynomial distribution controlled by a parameter $\eta_{c}$. The parameter $\eta_{c}$ controls how far the offspring individuals are generated away from its progenitors.

\subsection{Mutation Operator}

After the creation of offpring individuals, they are mutated in order to improve convergence and also to allow escaping from local optima. This mutation is performed with the Polynomial Mutation operator, successfully used in many problems and easily controlled by its mutation parameters $[69,70]$. The generation of a mutated individual $x$ from an offspring individual $c$ is also controlled by a polynomial probability distribution. Each variable $i$ of the mutated individual $x$ is created as

$$
x_{i}=\left\{\begin{array}{cl}
c_{i}+\delta_{L}\left(c_{i}-c_{i}^{(L)}\right), & \text { if } u \leq 0.5 \\
c_{i}+\delta_{R}\left(c_{i}^{(U)}-c_{i}\right), & \text { if } u>0.5
\end{array}\right.
$$

where $u$ is a random number generated within $[0,1]$, and $\delta_{L}$ and $\delta_{R}$ are the polynomial distributions, both controlled by a parameter $\eta_{m}$. Both distributions are modified, so that no value outside the specified range $\left[c_{i}^{(L)}, c_{i}^{(U)}\right]$ is created by this operator.

This operator generates individuals close to the offspring with a higher probability than individuals far away from it. This is done by considering that each variable $i$ of an offspring individual $c$ of length $p$ has a probability $1 / p$ of being mutated.

\subsection{Fitness Function}

The fitness function assigns a fitness value to each individual $x$ for each one of the objectives defined in a MOP. In our MOP, two objectives have been defined for the individual (10): (i) to minimize the error between the experimental values of $I_{D}\left(V_{G_{i}}, V_{D_{j}}\right)$ and their estimation from (1)-(2) $\widehat{I_{D}}\left(V_{G_{i}}, V_{D_{j}}, x\right)$, and (ii) to minimize the error between the voltage drops at the contact region $V_{S}\left(V_{G_{i}}, V_{D_{i}}, x\right)$ extracted from (2) and their estimation $\widehat{V_{S}}\left(V_{G_{i}}, V_{D_{j}}, x\right)$ extracted from (6).

The Normalized Root Mean Squared Error (NRMSE) is used to estimate these errors [71]:

$$
\operatorname{NRMSE}(y, \hat{y})=\sqrt{\frac{\sum_{z=1}^{w}\left(y_{z}-\hat{y}_{z}\right)^{2}}{\sum_{z=1}^{w}\left(y_{z}-\bar{y}\right)^{2}}}
$$

where $y$ represents the data set that we want to accurately approximate, $\hat{y}$ is the estimation of $y, w$ is the number of data samples in $y$, and $\bar{y}$ is the mean value of the complete data set $y$. Thus, the fitness function $f$ is defined as $f=\left(f_{1}, f_{2}\right)$, where

$$
\begin{aligned}
f_{1}(x)= & \operatorname{NRMSE}\left(I_{D}\left(V_{G_{i}}, V_{D_{j}}\right), \widehat{I_{D}}\left(V_{G_{i}}, V_{D_{j}}, x\right)\right), \\
f_{2}(x)= & \operatorname{NRMSE}\left(V_{S}\left(V_{G_{i}}, V_{D_{j}}, x\right), \widehat{V_{S}}\left(V_{G_{i}}, V_{D_{j}}, x\right)\right), \\
& \forall i \in \mathbb{Z}: 1 \leq i \leq g, \forall j \in \mathbb{Z}: 1 \leq j \leq d
\end{aligned}
$$

and

6.4.1. $I_{D}\left(V_{G_{i}}, V_{D_{j}}\right)$ $I_{D}\left(V_{G_{i}}, V_{D_{j}}\right)$ is the experimental value of the drain current;

\subsection{2. $\widehat{I_{D}}\left(V_{G_{i}}, V_{D_{j}}, x\right)$}

$\widehat{I_{D}}\left(V_{G_{i}}, V_{D_{j}}, x\right)$ is the estimation of $I_{D}\left(V_{G_{i}}, V_{D_{j}}\right)$ extracted from the compact model (1)-(2) using the bisection method [50]. This procedure starts with an initial interval $\left[a_{i}, b_{i}\right]$ in which the value of $\widehat{I_{D}}\left(V_{G_{i}}, V_{D_{j}}, x\right)$ is expected. A different interval may be defined for each value of $V_{G_{i}}$. This interval is shortened to the half in successive iterations until an adequate value for $\widehat{I_{D}}\left(V_{G_{i}}, V_{D_{j}}, x\right)$ is obtained.

Starting at iteration 0 , and assuming that $a_{i j_{0}}=a_{i}$ and $b_{i j_{0}}=b_{i}$, three values are considered in each iteration $t$ for all possible combinations of $i$ and $j$ :

$$
\begin{aligned}
& \widehat{I_{D_{a, t}}}\left(V_{G_{i}}, V_{D_{j}}, x\right)=a_{i j_{t}}, \\
& \widehat{I_{D_{b, t}}}\left(V_{G_{i}}, V_{D_{j}}, x\right)=b_{i j_{t}}, \\
& \widehat{I_{D_{c, t}}}\left(V_{G_{i}}, V_{D_{j}}, x\right)=c_{i j_{t}}=\left(a_{i j_{t}}+b_{i j_{t}}\right) / 2
\end{aligned}
$$

For each one of these values, $V_{S}$ is calculated using (2) and the different values of $\widehat{M_{C}}\left(V_{G_{i}}\right)$ coded in $x$ :

$$
\begin{gathered}
V_{S_{k, t}}\left(V_{G_{i}}, V_{D_{j}}, x\right)=\left(\frac{\widehat{I_{D_{k, t}}}\left(V_{G_{i}}, V_{D_{j}}\right)}{\widehat{M_{C}}\left(V_{G_{i}}\right)}\right)^{\frac{1}{m_{k}}}, \\
\forall i \in \mathbb{Z}: 1 \leq i \leq g, \forall j \in \mathbb{Z}: 1 \leq j \leq d, k \in\{a, b, c\}
\end{gathered}
$$

Then, the differences between each one of the three possible estimations $k \in\{a, b, c\}$ of $I_{D}$ in the current iteration and the value returned by the compact model (1) are calculated: 


$$
\begin{aligned}
r_{k, i j_{t}} & =\widehat{I_{D_{k, t}}}\left(V_{G_{i}}, V_{D_{j}}\right)+ \\
& +\frac{k^{\prime} W\left(V_{G_{i}}-V_{T}-V_{S_{k, t}}\left(V_{G_{i}}, V_{D_{j}}\right)\right)^{\gamma+2}}{L(\gamma+2)}- \\
& -\frac{k^{\prime} W\left(V_{G_{i}}-V_{T}-V_{D_{j}}\right)^{\gamma+2}}{L(\gamma+2)}
\end{aligned}
$$

As the bisection procedure tries to find a root in (18), the interval $\left[a_{i j_{t}}, b_{i j_{t}}\right]$ is shortened according to the values $r_{a, i j_{t}}, r_{b, i j_{t}}$ and $r_{c, i j_{t}}$ following the rule:

$$
\left[a_{i j_{t+1}}, b_{i j_{t+1}}\right]= \begin{cases}{\left[c_{i j_{t}}, b_{i j_{t}}\right],} & \text { if } \operatorname{sign}\left(r_{a, i j_{t}}\right)=\operatorname{sign}\left(r_{c, i j_{t}}\right) \\ {\left[a_{i j_{t}}, c_{i j_{t}}\right],} & \text { otherwise }\end{cases}
$$

This iterative process continues until the condition $\left|r_{c, i j_{t}}\right|<\varepsilon$ is reached or a maximum number of iterations are executed $\left(t=n_{\mathrm{bisec}}\right)$. In any case, the drain current is estimated as $\widehat{I_{D_{c, t}}}\left(V_{G_{i}}, V_{D_{j}}, x\right)=c_{i j_{t}}$.

\subsection{3. $V_{S}\left(V_{G_{i}}, V_{D_{j}}, x\right)$}

$V_{S}\left(V_{G_{i}}, V_{D_{j}}, x\right)$ is the voltage drop at the contact introducing in (2) the experimental value $I_{D}\left(V_{G_{i}}, V_{D_{j}}\right)$ and the parameter $\widehat{M_{C}}\left(V_{G_{i}}\right)$ coded in $x$ :

$$
V_{S}\left(V_{G_{i}}, V_{D_{j}}, x\right)=\left(\frac{I_{D}\left(V_{G_{i}}, V_{D_{j}}\right)}{\widetilde{M_{C}}\left(V_{G_{i}}\right)}\right)^{\frac{1}{m_{k}}}
$$

\subsection{4. $\widehat{V_{S}}\left(V_{G_{i}}, V_{D_{j}}, x\right)$}

$\widehat{V_{S}}\left(V_{G_{i}}, V_{D_{j}}, x\right)$ is the voltage drop at the contact introducing in (6) the experimental value $I_{D}\left(V_{G_{i}}, V_{D_{j}}\right)$ and the parameters $k^{\prime}, \gamma$ and $V_{T}$ coded in $x$ :

$$
\begin{aligned}
& \widehat{V_{S}}\left(V_{G_{i}}, V_{D_{j}}\right)=V_{G_{i}}-V_{T}- \\
& -\left[I_{D}\left(V_{G_{i}}, V_{D_{j}}\right) \frac{(\gamma+2) L}{W k^{\prime}}+\left(V_{G_{i}}-V_{T}-V_{D_{j}}\right)^{\gamma+2}\right]^{1 /(\gamma+2)}
\end{aligned}
$$

\section{Results}

In this section, two pairs of experiments $(A$ and $B$, and $C$ and $D$ ) are presented. These experiments have been carried out using an open source evolutionary tool called ECJ (A Javabased Evolutionary Computation Research System) [72]. Experiments $A$ and $B$ have been executed in a CentOS cluster with 19 computation nodes, each one with two Intel Xeon E5520 processors at $2.7 \mathrm{GHz}$. Each execution set for a determined $m_{k}$ was executed sequentially using only one node. Experiments $C$ and $D$ have been executed in an Ubuntu laptop with an Intel i5-3320M processor at $2.6 \mathrm{GHz}$.

In the experiments $A$ and $B$, the evolutionary parameter extraction procedure is applied to solve the problem presented at the end of Section II and Figs. 1 and 2, in which the $H_{V G}$ function was not provided with the appropriate input data. In experiment $A$ a parameter set is extracted from Fig. 1, while in experiment $B$ this extracted parameter set is optimized. Since the parameter set employed to generate the curves from Fig. 1
Table 1: NSGA-II execution parameters used in the different experiments.

\begin{tabular}{ccccc}
\hline & \multicolumn{4}{c}{ Experiment } \\
\cline { 2 - 5 } Parameter & $A$ & $B$ & $C$ & $D$ \\
\hline$N$ & 500 & 500 & 500 & 500 \\
$n_{j o b}$ & 10 & 10 & 10 & 10 \\
$n_{\text {iter }}$ & 50000 & 50000 & 5000 & 50000 \\
$p$ & 8 & 8 & 9 & 9 \\
$P_{m}$ & 0.125 & 0.125 & 0.1111 & 0.1111 \\
$P_{c}$ & 0.5 & 0.5 & 0.5 & 0.5 \\
$\eta_{c}$ & 20 & 20 & 20 & 20 \\
$\eta_{m}$ & 20 & 20 & 20 & 20 \\
\hline
\end{tabular}

is known, it can be used along with the fulfillment of $f(x)(15)$ to verify the validity of the evolutionary procedure as a parameter extraction method in OTFTs. Experiments $C$ and $D$, on the other hand, deal with the optimization and extraction of parameters of two real OTFT devices [17, 73]. In Experiment $C$, a solution for the parameter set is also known [17], which can be compared against our results to test the effectiveness of our procedure.

\subsection{Experiment A: Parameter Extraction}

Once the set of experimental data is available (curves in Fig. 1), the parameters of the evolutionary procedure are defined.

First. As seen in Fig. $1, V_{D}$ is swept from 0 to $-60 \mathrm{~V}$ with a $-2.4 \mathrm{~V}$ step and $V_{G}$ is swept from 0 to $-50 \mathrm{~V}$ with a $-12.5 \mathrm{~V}$ step. That is, $V_{G_{1}}=0 \mathrm{~V}, V_{G_{2}}=-12.5 \mathrm{~V}, V_{G_{3}}=-25 \mathrm{~V}$, $V_{G_{4}}=-37.5 \mathrm{~V}$ and $V_{G_{2}}=-50 \mathrm{~V}$.

Second. The search space is defined in order to guide the search to an optimum solution. For this problem, the parameter ranges of each individual $x$ of the population are:

$$
\begin{aligned}
k^{\prime} & \in\left[1.0 \times 10^{-16}, 1.0 \times 10^{-10}\right] \mathrm{A} / \mathrm{V}^{(2+\gamma)} \\
\gamma & \in[0,2] \\
V_{T} & \in[-30,30] \mathrm{V} \\
\widehat{M_{C}}\left(V_{G_{i}}\right) & \in\left[1.0 \times 10^{-10}, 1.0 \times 10^{-4}\right] \mathrm{A} / \mathrm{V}^{m_{k}}
\end{aligned}
$$

As justified in Section 6.A, the parameter $m_{k}$ is considered a static predefined parameter. Eleven cases are analyzed, in which $m_{k}$ is swept from 1.0 to 2.0 with a 0.1 step.

Third. Since EAs are metaheuristic, each execution of our evolutionary procedure to extract the device parameters may return a different result, thereby the NSGA-II is executed $n_{j o b}$ times for every $m_{k}$ value, delimiting each one of them to $n_{\text {iter }}$ iterations, being the initial $N$ individuals randomly generated. In order to define the value of $N, n_{j o b}$ and $n_{\text {iter }}$ (Table 1) several trial-and-error tests are carried out. A compromise between the quality of the result and the computational cost needed to obtain it must be achieved. We observed that increasing the value of 
Table 2: Bisection method parameters used in the different experiments.

\begin{tabular}{cccc}
\hline & \multicolumn{3}{c}{ Experiment } \\
\cline { 2 - 4 } Param. & $A$ and $B$ & $C$ & $D$ \\
\hline$\varepsilon$ & $1.0 \times 10^{-15}$ & $1.0 \times 10^{-15}$ & $1.0 \times 10^{-15}$ \\
$n_{\text {bisec }}$ & 1000 & 1000 & 1000 \\
{$\left[a_{i}, b_{i}\right]$} & {$\left[0,-1.0 \times 10^{-6}\right]$} & {$\left[0,-1.0 \times 10^{-8}\right]$} & {$\left[0,-1.0 \times 10^{-6}\right]$} \\
\hline
\end{tabular}

Table 3: Mean \pm standard deviation of $f_{1}$ and $f_{2}$ objectives for each Pareto front related to a different $m_{k}$ value.

\begin{tabular}{|c|c|c|}
\hline$m_{k}$ & $f_{1}$ & $f_{2}$ \\
\hline 1.0 & $1.18 \times 10^{-2} \pm 8.0 \times 10^{-4}$ & $3.38 \times 10^{-1} \pm 7.06 \times 10^{-2}$ \\
\hline 1.1 & $2.98 \times 10^{0} \pm 2.2 \times 10^{+1}$ & $3.42 \times 10^{-1} \pm 1.24 \times 10^{-1}$ \\
\hline 1.2 & $7.00 \times 10^{-4} \pm 1.0 \times 10^{-4}$ & $9.75 \times 10^{-1} \pm 5.19 \times 10^{-1}$ \\
\hline 1.3 & $1.19 \times 10^{-2} \pm 4.0 \times 10^{-3}$ & $4.35 \times 10^{-1} \pm 2.07 \times 10^{-1}$ \\
\hline 1.4 & $1.26 \times 10^{-2} \pm 4.1 \times 10^{-3}$ & $1.51 \times 10^{0} \pm 0.84 \times 10^{-1}$ \\
\hline 1.5 & $1.20 \times 10^{-2} \pm 4.2 \times 10^{-3}$ & $1.72 \times 10^{0} \pm 1.07 \times 10^{0}$ \\
\hline 1.6 & $6.20 \times 10^{-3} \pm 3.9 \times 10^{-3}$ & $2.16 \times 10^{0} \pm 1.19 \times 10^{0}$ \\
\hline 1.7 & $1.01 \times 10^{-2} \pm 6.3 \times 10^{-3}$ & $1.76 \times 10^{0} \pm 9.73 \times 10^{-1}$ \\
\hline 1.8 & $1.19 \times 10^{-2} \pm 6.3 \times 10^{-3}$ & $1.37 \times 10^{0} \pm 7.20 \times 10^{-1}$ \\
\hline 1.9 & $5.30 \times 10^{-3} \pm 3.7 \times 10^{-3}$ & $2.24 \times 10^{0} \pm 1.16 \times 10^{0}$ \\
\hline 2.0 & $1.08 \times 10^{-2} \pm 8.9 \times 10^{-3}$ & $1.86 \times 10^{0} \pm 1.01 \times 10^{0}$ \\
\hline
\end{tabular}

the parameters $N$ and $n_{\text {iter }}$ accurate results are obtained. However, it increases considerably the computational cost and in addition, the increment of the values of $N$ or $n_{\text {iter }}$ does not always ensure a quality increment of the final result, since for example the evolutionary procedure might converge to a local optimum. In those cases in which the evolutionary procedure always tends to converge to a local optimum, the mutation operators will help escaping from it, but with an increased execution time.

The values of $\eta_{c}$ and $\eta_{m}$ (Table 1) are standard values that have been tested successfully, and also incorporated in the ECJ evolutionary tool.

Fourth. The parameters needed to carry out the bisection method for the computation of the fitness function are presented in Table 2. For this experiment, we use the same initial interval $\left[a_{i}, b_{i}\right]$ for each $V_{G_{i}}$, in which the bisection method converges successfully.

Fifth. The evolutionary parameter extraction procedure is finally executed. At the end of the evolutionary process execution, a set of $n_{j o b}$ Pareto fronts are obtained for every $m_{k}$ value. In order to discard non-optimum solutions, the expert or final user should choose the best Pareto front associated with each $m_{k}$ value, discarding the remaining $\left(n_{j o b}-1\right)$ Pareto fronts. At this point, there should be as many Pareto fronts as different $m_{k}$ values are available, being each one of them associated with its own $m_{k}$ value. In order to have an idea about the distribution of the different Pareto-optimum solutions, their mean and standard

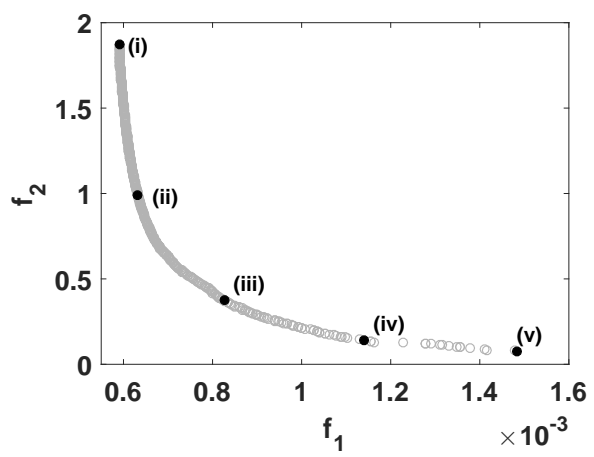

Figure 4: Pareto front selected as best result of the evolutionary procedure execution. It corresponds to $m_{k}=1.2$. The parameters of the Pareto-optimum solutions enumerated from $(i)$ to $(v)$ and marked in black are found in Table 5. The current-voltage curves corresponding to these five solutions are represented in Fig. 5.

Table 4: Mean \pm standard deviation of the estimated parameters which compose the Pareto front represented in Fig. 4. $\left(k^{\prime}\right.$ is in $\mathrm{A} / \mathrm{V}^{2+\gamma}, V_{T}$ in $\mathrm{V}$ and $\widehat{M_{C}}\left(V_{G}\right)$ in $\left.\mathrm{A} / \mathrm{V}^{m_{k}}\right)$

\begin{tabular}{cc}
\hline$x$ & Value \\
\hline$k^{\prime}$ & $1.59 \times 10^{-14} \pm 8.84 \times 10^{-29}$ \\
$\gamma$ & $9.65 \times 10^{-1} \pm 1.50 \times 10^{-3}$ \\
$V_{T}$ & $9.55 \times 10^{0} \pm 2.07 \times 10^{-2}$ \\
$\widehat{M_{C}}(0 \mathrm{~V})$ & $9.86 \times 10^{-5} \pm 2.63 \times 10^{-6}$ \\
$\widehat{M}_{C}(-12.5 \mathrm{~V})$ & $1.24 \times 10^{-6} \pm 8.88 \times 10^{-7}$ \\
$\widehat{M}_{C}(-25 \mathrm{~V})$ & $9.90 \times 10^{-7} \pm 4.28 \times 10^{-7}$ \\
$\widehat{M}_{C}(-37.5 \mathrm{~V})$ & $2.21 \times 10^{-6} \pm 1.17 \times 10^{-6}$ \\
$\widehat{M}_{C}(-50 \mathrm{~V})$ & $8.65 \times 10^{-6} \pm 8.54 \times 10^{-6}$ \\
\hline
\end{tabular}

deviation are calculated and shown in Table 3. Table 3 shows that in most of the cases the procedure finds accurate solutions for $f_{1}$ while the results for $f_{2}$ cannot be considered as accurate as expected. Analyzing the mean and standard deviation values calculated for the $f_{2}$ objective, the $m_{k}$ cases from 1.4 to 2.0 (both included) are discarded as possible search starting points because of their high $f_{2}$ mean and standard deviation values. The $m_{k}=1.1$ case is also discarded because the $f_{1}$ mean and standard values are not as accurate as expected. All the rest $m_{k}$ cases have similar $f_{2}$ mean values. Among these three last cases, $m_{k}=1.2$ is the best option as the search starting point, since it has the most accurate $f_{1}$ mean value. In order to confirm whether this decision is correct or not, we use graphical representations and distributed statistics to study the obtained parameter sets. The Pareto front associated with the chosen $m_{k}$ value is represented in Fig. 4 while the statistics associated with its estimated parameter sets are represented in Table 4.

Since the Pareto front from Fig. 4 is composed by too many Pareto-optimum solutions, only five of them are selected (enumerated black points in Fig. 4) (note that all the Paretooptimum solutions that compose a determined Pareto front are 
Table 5: Estimated parameters of the Pareto-optimum solutions marked with black points in Fig. 4 and represented in Fig. $5\left(k^{\prime}\right.$ is in $\mathrm{A} / \mathrm{V}^{2+\gamma}, V_{T}$ in $\mathrm{V}$ and $\widehat{M_{C}}\left(V_{G}\right)$ in $\mathrm{A} / \mathrm{V}^{m_{k}}$ ).

\begin{tabular}{cccccc}
\hline & \multicolumn{5}{c}{ Pareto-optimum Solutions } \\
\cline { 2 - 6 }$x$ & $(i)$ & $(i i)$ & $($ iii $)$ & $(i v)$ & $(v)$ \\
\hline$k^{\prime}$ & $1.59 \times 10^{-14}$ & $1.59 \times 10^{-14}$ & $1.59 \times 10^{-14}$ & $1.59 \times 10^{-14}$ & $1.59 \times 10^{-14}$ \\
$\gamma$ & $9.63 \times 10^{-1}$ & $9.64 \times 10^{-1}$ & $9.68 \times 10^{-1}$ & $9.68 \times 10^{-1}$ & $9.67 \times 10^{-1}$ \\
$V_{T}$ & $9.54 \times 10^{0}$ & $9.54 \times 10^{0}$ & $9.52 \times 10^{0}$ & $9.59 \times 10^{0}$ & $9.65 \times 10^{0}$ \\
$\widehat{M}_{C}(0 V)$ & $9.96 \times 10^{-5}$ & $9.93 \times 10^{-5}$ & $9.99 \times 10^{-5}$ & $9.90 \times 10^{-5}$ & $9.99 \times 10^{-5}$ \\
$\widehat{M}_{C}(-12.5 \mathrm{~V})$ & $3.43 \times 10^{-6}$ & $1.09 \times 10^{-6}$ & $3.50 \times 10^{-7}$ & $1.78 \times 10^{-7}$ & $1.34 \times 10^{-7}$ \\
$\widehat{M}_{C}(-25 \mathrm{~V})$ & $1.75 \times 10^{-6}$ & $9.94 \times 10^{-7}$ & $4.44 \times 10^{-7}$ & $3.18 \times 10^{-7}$ & $2.88 \times 10^{-7}$ \\
$\widehat{M}_{C}(-37.5 \mathrm{~V})$ & $4.48 \times 10^{-6}$ & $2.06 \times 10^{-6}$ & $7.75 \times 10^{-7}$ & $5.96 \times 10^{-7}$ & $5.78 \times 10^{-7}$ \\
$\widehat{M}_{C}(-50 \mathrm{~V})$ & $3.19 \times 10^{-5}$ & $5.26 \times 10^{-6}$ & $1.36 \times 10^{-6}$ & $1.05 \times 10^{-6}$ & $1.08 \times 10^{-6}$ \\
\hline
\end{tabular}

indifferent to each other and equally valid). The $I_{D}-V_{D}$ and $I_{D}-V_{C}$ curves associated to these five solutions are represented in Fig. 5 . As we can see in Fig. 4, each Pareto-optimum solution returns a different couple of values for the fitness function $f=\left(f_{1}, f_{2}\right)$. If we explore the different Pareto-optimum solutions from left to right along their Pareto front, the $f_{1}$ values worsen while the $f_{2}$ values improve. Solution $(i)$ has the best $f_{1}$ value (minimization), however it has the worst $f_{2}$ value of the Pareto front. In the opposite case, solution (v) has the best $f_{2}$ value but the worst $f_{1}$ value of the complete Pareto-optimum solution set.

In order to confirm the differences between the different Pareto-optimum solutions of the Pareto front, the estimated parameters of the Pareto-optimum solutions from Fig. 5 are represented in Table 5. This table shows that the different values of the parameters $k^{\prime}, \gamma$ and $V_{T}$ are very close to each other. This fact along with the good agreement shown with the $I_{D}-V_{D}$ curves help us to confirm that in these cases the evolutionary procedure converges. However, the solutions (i), (ii) and (iii) provide a poor agreement with the $I_{D}-V_{C}$ curves, which is directly related with $\widehat{M_{C}}\left(V_{G}\right)$. Note that both positive and negative values for the contact voltage are obtained at low currents for the (i), (ii) and (iii) cases of Fig. 5, while only negative values are allowed.

Since all the solutions are considered equivalent in the Pareto-optimum solution set, an expert opinion is compulsory. Based on our expert judgment, together with the facts exposed previously, solution $(v)$ with $f=\left(1.48 \times 10^{-3}, 7.83 \times 10^{-2}\right)$ has been selected as final solution. This solution is represented in Fig. 5 and its estimated parameter set is shown in Table 6 along with its relative error (RE). Comparing the values of the estimated parameter set shown in Table 6 and the original values, we conclude that the procedure is able to find accurate values for $V_{T}$ and $\gamma$, solving the problem that appears when the $H_{V G}$ function is provided with an improper set of experimental data. Also, the extracted value of $k^{\prime}$ is considered as valid. However the $\overline{M_{C}}\left(V_{G}\right)$ set differs considerably from the $M_{C}\left(V_{G}\right)$ set despite a good agreement with the $I_{D}-V_{S}$ curves is apparently
Table 6: Final solution of the evolutionary procedure, original parameter set employed and the RE of the chosen final solution, $(v)$ in Fig. $5\left(k^{\prime}\right.$ is in $\mathrm{A} / \mathrm{V}^{2+\gamma}$, $V_{T}$ in $\mathrm{V}$ and $\widehat{M_{C}}\left(V_{G}\right)$ in $\left.\mathrm{A} / \mathrm{V}^{m_{k}}\right)$.

\begin{tabular}{cllc}
\hline$x$ & Original Value & Estimated Value & $\mathrm{RE}(\%)$ \\
\hline$k^{\prime}$ & $1.38 \times 10^{-14}$ & $1.59 \times 10^{-14}$ & $1.57 \times 10^{+1}$ \\
$\gamma$ & $1.00 \times 10^{0}$ & $9.67 \times 10^{-1}$ & $3.22 \times 10^{0}$ \\
$V_{T}$ & $1.00 \times 10^{+1}$ & $9.65 \times 10^{0}$ & $3.40 \times 10^{0}$ \\
$\widehat{M}_{C}(0 \mathrm{~V})$ & $5.00 \times 10^{-8}$ & $9.99 \times 10^{-5}$ & $1.99 \times 10^{+5}$ \\
$\widehat{M}_{C}(-12.5 \mathrm{~V})$ & $2.53 \times 10^{-7}$ & $1.34 \times 10^{-7}$ & $4.70 \times 10^{+1}$ \\
$\widehat{M}_{C}(-25 \mathrm{~V})$ & $6.12 \times 10^{-7}$ & $2.88 \times 10^{-7}$ & $5.28 \times 10^{+1}$ \\
$\widehat{M}_{C}(-37.5 \mathrm{~V})$ & $1.12 \times 10^{-6}$ & $5.78 \times 10^{-7}$ & $4.87 \times 10^{+1}$ \\
$\widehat{M}_{C}(-50 \mathrm{~V})$ & $1.80 \times 10^{-6}$ & $1.08 \times 10^{-6}$ & $3.97 \times 10^{+1}$ \\
\hline
\end{tabular}

obtained.

A further study of Fig. 4 shows that the obtained solutions are not spread evenly along the Pareto front, observing empty areas. This fact together with the low quality of some solutions (reasons exposed previously for solutions (i), (ii) and (iii)), make us think that a new search around a better known starting point could help us to get to a more accurate parameter set. In the next section, the evolutionary procedure is again executed, but this time using solution $(v)$ as initial starting point, and applying the evolutionary procedure as optimization method.

\subsection{Experiment B: Parameter Optimization}

The use of the evolutionary procedure as an optimization method implies to work with an initial set of values for the fitting parameters. The search space domain must be defined around these initial values. The previous analysis showed that the $f_{2}$ function is more sensitive to variations of the fitting parameters, i.e. the $I_{D}-V_{S}$ curves are more sensitive than the $I_{D}-V_{D}$ curves to variations in the transistor parameters, in particular the $M_{C}$ set. Then, the search space domains for the $k^{\prime}$, $\gamma$ and $V_{T}$ parameters are reduced, but not for the $\widehat{M_{C}}\left(V_{G}\right)$ set. 

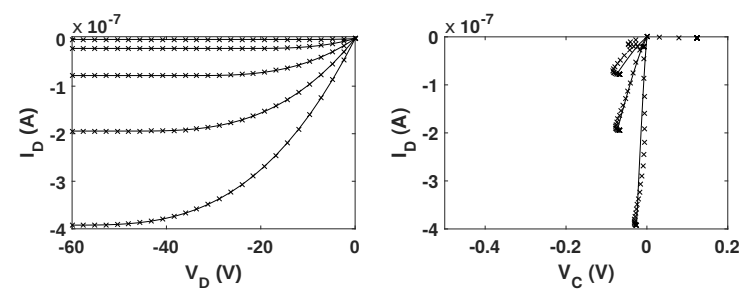

(i) $f=\left(5.90 \times 10^{-4}, 1.86\right)$
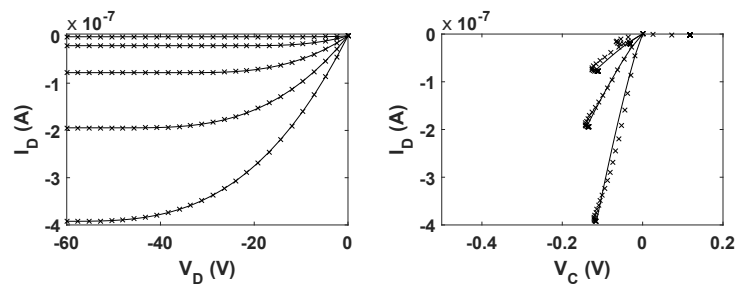

(ii) $f=\left(6.32 \times 10^{-4}, 9.9 \times 10^{-1}\right)$
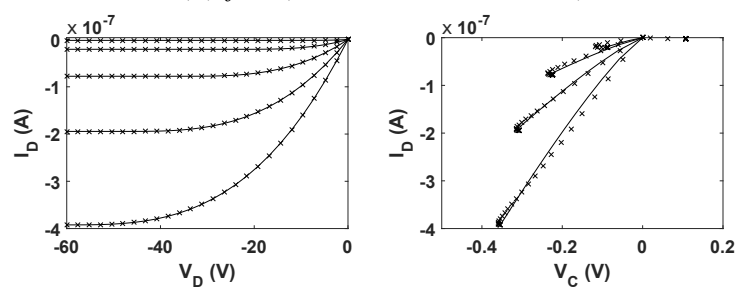

(iii) $f=\left(8.26 \times 10^{-4}, 3.78 \times 10^{-1}\right)$
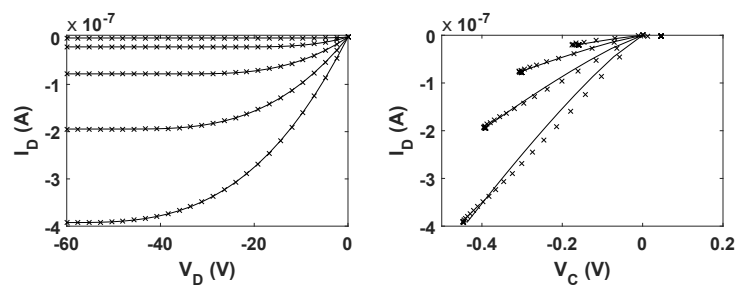

(iv) $f=\left(1.14 \times 10^{-3}, 1.41 \times 10^{-1}\right)$
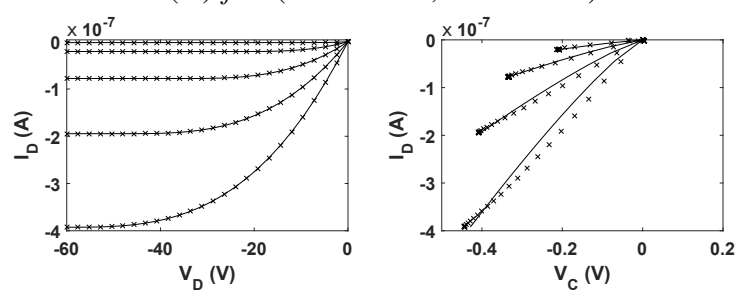

(v) $f=\left(1.48 \times 10^{-3}, 7.83 \times 10^{-2}\right)$

Figure 5: Output characteristics and contact $I-V$ curves for the five Paretooptimum solutions marked in Fig. 4. $I_{D}$ and $V_{C}$ are represented with symbols and $\widehat{I_{D}}$ and $\widehat{V_{C}}$ in solid lines. $V_{G}$ is swept from 0 to $-50 \mathrm{~V}$ with a $-12.5 \mathrm{~V}$ step (from top to bottom in each figure).

This will prevent the search from falling into a local optimum solution. The value of $m_{k}$ is also fixed to 1.2 (as above). Thus, the parameter ranges are

$$
\begin{aligned}
k^{\prime} & \in\left[10^{-14}, 10^{-13}\right] \mathrm{A} / \mathrm{V}^{(2+\gamma)} \\
\gamma & \in[0.8,1.1] \\
V_{T} & \in[8.5,1.1] \mathrm{V} \\
\widehat{M_{C}}\left(V_{G_{i}}\right) & \in\left[10^{-10}, 10^{-4}\right] \mathrm{A} / \mathrm{V}^{m_{k}}
\end{aligned}
$$

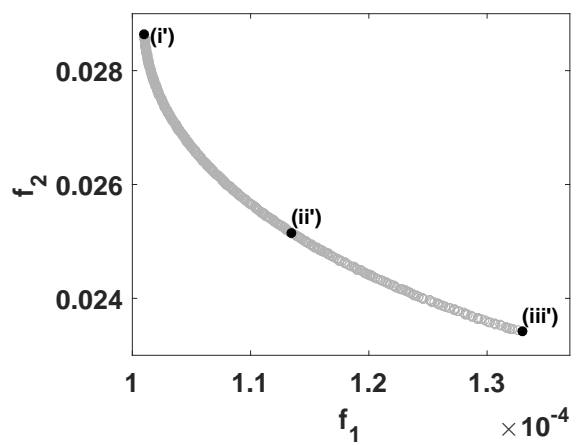

Figure 6: Pareto front selected as best result of the optimization execution. The parameters of the Pareto-optimum solutions enumerated from ( $\left.i^{\prime}\right)$ to (iii') and marked in black are found in Table 8 . The current-voltage curves corresponding to these three solutions are represented in Fig. 7.

Table 7: Mean \pm standard deviation of the estimated parameters which compose the Pareto-optimum solutions in Fig. $6\left(k^{\prime}\right.$ is in $\mathrm{A} / \mathrm{V}^{2+\gamma}, V_{T}$ in $\mathrm{V}$ and $\widehat{M_{C}}\left(V_{G}\right)$ in $\mathrm{A} / \mathrm{V}^{m_{k}}$ ).

\begin{tabular}{cc}
\hline$x$ & Value \\
\hline$k^{\prime}$ & $1.41 \times 10^{-14} \pm 1.40 \times 10^{-21}$ \\
$\gamma$ & $9.93 \times 10^{-1} \pm 3.55 \times 10^{-10}$ \\
$V_{T}$ & $9.91 \times 10^{0} \pm 2.97 \times 10^{-3}$ \\
$\widehat{M_{C}}(0 \mathrm{~V})$ & $1.32 \times 10^{-7} \pm 1.65 \times 10^{-8}$ \\
$\widehat{M}_{C}(-12.5 \mathrm{~V})$ & $2.88 \times 10^{-7} \pm 8.96 \times 10^{-9}$ \\
$\widehat{M}_{C}(-25 \mathrm{~V})$ & $6.41 \times 10^{-7} \pm 1.25 \times 10^{-8}$ \\
$\widehat{M}_{C}(-37.5 \mathrm{~V})$ & $1.19 \times 10^{-6} \pm 1.77 \times 10^{-8}$ \\
$\widehat{M}_{C}(-50 \mathrm{~V})$ & $1.98 \times 10^{-6} \pm 2.41 \times 10^{-8}$ \\
\hline
\end{tabular}

Once the evolutionary procedure is executed with these new parameter ranges, a new set of $n_{j o b}$ Pareto fronts is obtained, and the best one is selected (Fig. 6). The mean and standard deviation of the estimated parameters of the Pareto-optimum solutions of Fig. 6 are shown in Table 7. The optimization execution improves clearly the previous solution, confirming that the evolutionary procedure helps to optimize previous OTFT parameter sets by exploring the search space around them. In particular, there is a noticeable improvement in the standard deviation of the $\widehat{M_{C}}\left(V_{G}\right)$ set (compare Tables 4 and 7 ). This improvement is also seen in the current-voltage curves generated with the new solution.

As it happened before, the Pareto Front of Fig. 6 is composed by many Pareto-optimum solutions. Once again, the graphical representation of some of the solutions helps to select the best one. The current-voltage curves of three solutions extracted from Fig. 6 (black points and enumerated from (i') to (iii')) are represented in Fig. 7. Their estimated parameters are found in Table 8 . Following the expert criteria and examining the information provided by Table 8 and Fig. 7 , solution $(i ')$ is selected as the final solution of the evolutionary procedure, with $f=\left(1.01 \times 10^{-4}, 2.86 \times 10^{-2}\right)$ (along with 

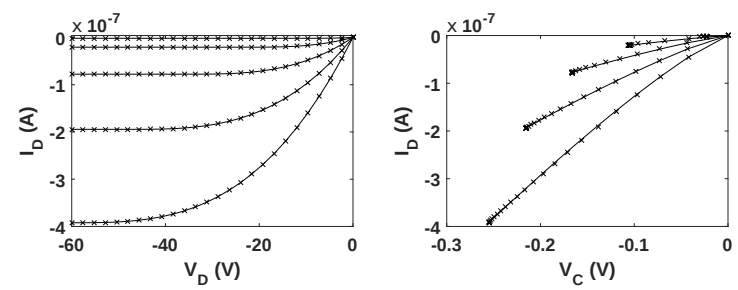

(i') $f=\left(1.01 \times 10^{-4}, 2.86 \times 10^{-2}\right)$
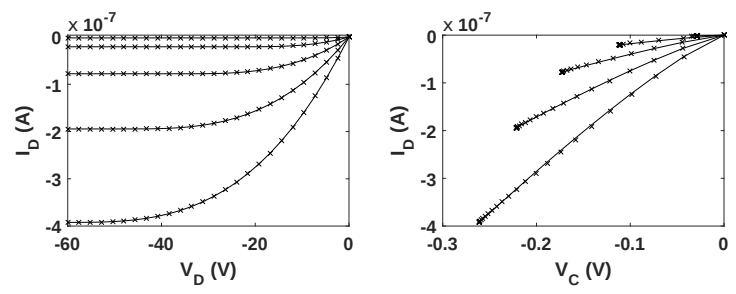

(ii') $f=\left(1.13 \times 10^{-4}, 2.51 \times 10^{-2}\right)$
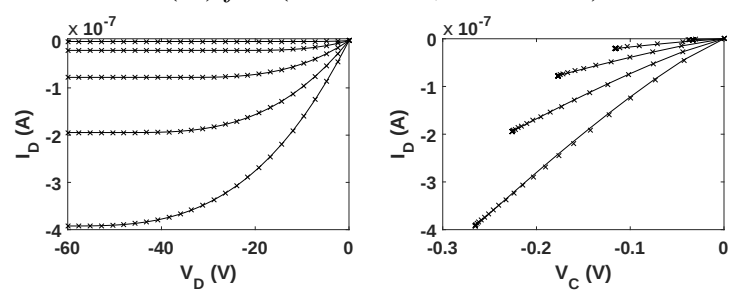

(iii') $f=\left(1.32 \times 10^{-04}, 2.34 \times 10^{-2}\right)$

Figure 7: Output characteristics and contact $I-V$ curves for the three Paretooptimum solutions marked in Fig. 6. $I_{D}$ and $V_{C}$ are represented with symbols and $\widehat{I_{D}}$ and $\widehat{V_{C}}$ in solid lines. $V_{G}$ is swept from 0 to $-50 \mathrm{~V}$ with a $-12.5 \mathrm{~V}$ step (from top to bottom in each figure).

$\left.m_{k}=1.2\right)$. The estimated parameter set of this solution is represented in Table 9 along with the original parameter set and its RE. If Tables 6 and 9 are compared, the solution in Table 9 is closer to the original parameter set than the one in Table 6 . The improvement shown in Table 9 is confirmed in the representation of the output characteristics and contact current-voltage curves of Fig. 7, in which a better agreement between experimental and estimated curves is observed. These facts confirm the success of the evolutionary procedure to optimize a previous solution.

\subsection{Experimental Application}

In the preceding sections, our evolutionary procedure has been successfully applied as a parameter extraction and as an optimization method to $I_{D}-V_{D}$ curves generated with model (1). In this section, these parameter optimization and parameter extraction methods are tested with two sets of experimental data measured in different OTFTs.

\subsubsection{Experiment C: Parameter Optimization}

The objective of this subsection is to optimize previously extracted parameters of an octadecyl substituted copper tetrabenzotriazaporphyrin (10CuTBTAP) based OTFT [17]. This bottom-gate, bottom-contact OTFT was fabricated on octadecyltrichlorosilane treated 250-nm-thick $\mathrm{SiO}_{2}$ gate insulator on the highly doped (resistivity 1 to $5 \Omega \mathrm{cm}$ ) Si(110) substrate. A
Table 8: Estimated parameters of the Pareto-optimum solutions marked with black points in Fig. 6 and represented in Fig. $7\left(k^{\prime}\right.$ is in $\mathrm{A} / \mathrm{V}^{2+\gamma}, V_{T}$ in $\mathrm{V}$ and $\widehat{M_{C}}\left(V_{G}\right)$ in $\left.\mathrm{A} / \mathrm{V}^{m_{k}}\right)$

\begin{tabular}{cccc}
\hline & \multicolumn{3}{c}{ Pareto-optimum Solutions } \\
\cline { 2 - 4 }$x$ & $(i)$ & $(\mathrm{ii})$ & $(\mathrm{iii})$ \\
\hline$k^{\prime}$ & $1.41 \times 10^{-14}$ & $1.41 \times 10^{-14}$ & $1.41 \times 10^{-14}$ \\
$\gamma$ & $9.93 \times 10^{-1}$ & $9.93 \times 10^{-1}$ & $9.93 \times 10^{-1}$ \\
$V_{T}$ & $9.91 \times 10^{0}$ & $9.91 \times 10^{0}$ & $9.92 \times 10^{0}$ \\
$\widehat{M_{C}}(0 \mathrm{~V})$ & $1.63 \times 10^{-7}$ & $1.23 \times 10^{-7}$ & $1.04 \times 10^{-7}$ \\
$\widehat{M_{C}}(-12.5 \mathrm{~V})$ & $3.03 \times 10^{-7}$ & $2.84 \times 10^{-7}$ & $2.72 \times 10^{-7}$ \\
$\widehat{M_{C}}(-25 \mathrm{~V})$ & $6.61 \times 10^{-7}$ & $6.34 \times 10^{-7}$ & $6.16 \times 10^{-7}$ \\
$\widehat{M_{C}}(-37.5 \mathrm{~V})$ & $1.22 \times 10^{-6}$ & $1.18 \times 10^{-6}$ & $1.15 \times 10^{-6}$ \\
$\widehat{M_{C}}(-50 \mathrm{~V})$ & $2.01 \times 10^{-6}$ & $1.96 \times 10^{-6}$ & $1.93 \times 10^{-6}$ \\
\hline
\end{tabular}

Table 9: Final solution of the optimization execution, original parameter set employed and RE of the chosen final solution, $(i)$ in Fig. $7\left(k^{\prime}\right.$ is in $\mathrm{A} / \mathrm{V}^{2+\gamma}$, $V_{T}$ in $\mathrm{V}$ and $\widehat{M_{C}}\left(V_{G}\right)$ in $\left.\mathrm{A} / \mathrm{V}^{m_{k}}\right)$.

\begin{tabular}{clll}
\hline$x$ & Original Value & Estimated Value & RE $(\%)$ \\
\hline$k^{\prime}$ & $1.38 \times 10^{-14}$ & $1.41 \times 10^{-14}$ & $2.66 \times 10^{0}$ \\
$\gamma$ & $1.00 \times 10^{0}$ & $9.93 \times 10^{-1}$ & $6.18 \times 10^{-1}$ \\
$V_{T}$ & $1.00 \times 10^{1}$ & $9.91 \times 10^{0}$ & $8.71 \times 10^{-1}$ \\
$\widehat{M_{C}}(0 \mathrm{~V})$ & $5.00 \times 10^{-8}$ & $1.63 \times 10^{-7}$ & $2.26 \times 10^{2}$ \\
$\widehat{M}_{C}(-12.5 \mathrm{~V})$ & $2.53 \times 10^{-7}$ & $3.03 \times 10^{-7}$ & $2.00 \times 10^{1}$ \\
$\widehat{M}_{C}(-25 \mathrm{~V})$ & $6.12 \times 10^{-7}$ & $6.61 \times 10^{-7}$ & $8.01 \times 10^{0}$ \\
$\widehat{M}_{C}(-37.5 \mathrm{~V})$ & $1.12 \times 10^{-6}$ & $1.22 \times 10^{-6}$ & $8.21 \times 10^{0}$ \\
$\widehat{M}_{C}(-50 \mathrm{~V})$ & $1.80 \times 10^{-6}$ & $2.01 \times 10^{-6}$ & $1.22 \times 10^{1}$ \\
\hline
\end{tabular}

70-nm-thick film of 10CuTBTAP was spin-coated onto photolithographically prepatterned 200-nm-thick gold source-drain electrodes in an interdigitated configuration with channel width $W=2 \mathrm{~mm}$ and length $L=5 \mu \mathrm{m}$. The full protocols of the electrode deposition, substrate cleaning, and surface passivation were given in [17]. The electrical measurements were performed at room temperature in air under ambient conditions.

The experimental $I_{D}-V_{D}$ curves of this transistor are represented with crosses in Fig. 8a. The optimization procedure defined for experiment $C$ is applied to the experimental data of Fig. 8a. The NSGA-II execution parameters are shown in Table 1 and the parameters of the bisection method in Table 2. The parameter set extracted in [17] is shown in Table 10 and is considered as the initial previous solution in the optimization procedure. In this case, $m_{k}=1.0$ and the range defined for each parameter is 


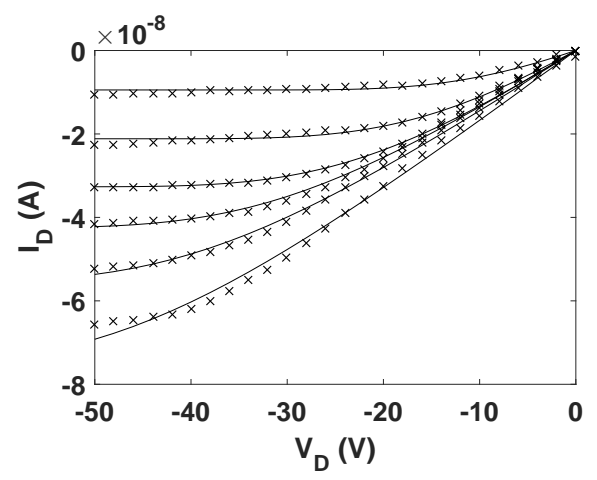

(a)

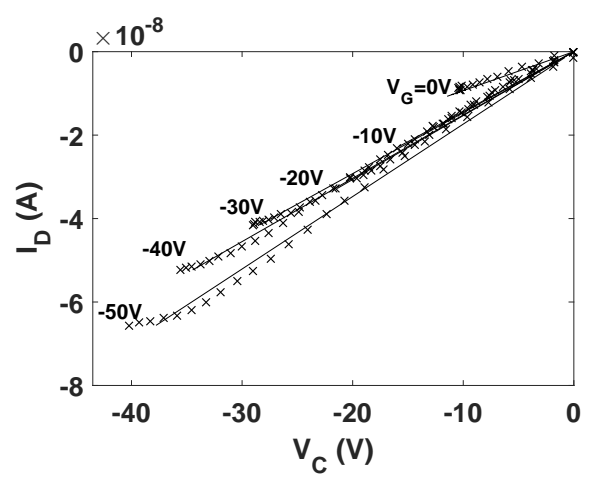

(b)

Figure 8: Experiment C. (a) Comparison of experimental output characteristics $(\times)$ and the numerical evaluation of (1) incorporating the estimated parameter set (solid lines) at different gate voltages. (b) Current-voltage curves at the contact extracted from (6) using the experimental output characteristics at different gate voltages $(\times)$. The solid lines are the fittings using (2) and the estimated parameter set. In (a) and (b) $V_{G}$ is swept from 0 to $-50 \mathrm{~V}$ with a $-10.0 \mathrm{~V}$ step (from top to bottom).

$$
\begin{aligned}
k^{\prime} & \in\left[2.5 \times 10^{-17}, 2.5 \times 10^{-15}\right] \mathrm{A} / \mathrm{V}^{(2+\gamma)} \\
\gamma & \in[1.0,2.0] \\
V_{T} & \in[25,45] \mathrm{V} \\
\widehat{M_{C}}\left(V_{G_{i}}\right) & \in\left[10^{8}, 2.0 \times 10^{9}\right] \mathrm{A} / \mathrm{V}^{m_{k}}
\end{aligned}
$$

Once the evolutionary procedure is executed, the different Pareto fronts are analyzed and the best solution (following an expert criteria) is selected. The values of the parameters which compose the chosen solution are shown in Table 10 (optimized solution). The fitness function of the result obtained in [17] is $f=\left(6.58 \times 10^{-2}, 9.92 \times 10^{-2}\right)$ while the fitness function obtained with the evolutionary procedure is $f=\left(5.4 \times 10^{-2}, 7.1 \times 10^{-2}\right)$. This means an improvement over the previous solution of $18.15 \%$ for the $f_{1}$ objective $\left(I_{D}-V_{D}\right.$ agreement) and $28 \%$ for the $f_{2}$ objective $\left(I_{D}-V_{S}\right.$ agreement). Although these percentages may not seem large enough, the improvement on the values of the transistor parameters is enormous. Note simply the variation in the threshold voltage of around $9 \mathrm{~V}$ from an initial value of $43 \mathrm{~V}$. This optimization effort means a great advance in the accurate characterization of OTFTs.
Table 10: Parameter values of the previous and optimized solutions of the 10CuTBTAP OTFT $\left(k^{\prime}\right.$ is in A/ $\mathrm{V}^{2+\gamma}, V_{T}$ in $\mathrm{V}$ and $\widehat{M_{C}}\left(V_{G}\right)$ in $\left.\mathrm{A} / \mathrm{V}^{m_{k}}\right)$.

\begin{tabular}{ccc}
\hline$x$ & $\begin{array}{c}\text { Previous } \\
\text { Solution [17] }\end{array}$ & $\begin{array}{c}\text { Optimized } \\
\text { Solution }\end{array}$ \\
\hline$k^{\prime}$ & $2.98 \times 10^{-16}$ & $2.75 \times 10^{-16}$ \\
$\gamma$ & $1.63 \times 10^{0}$ & $1.99 \times 10^{0}$ \\
$V_{T}$ & $4.32 \times 10^{+1}$ & $3.43 \times 10^{+1}$ \\
$\widehat{M_{C}}(0 \mathrm{~V})$ & $1.13 \times 10^{+9}$ & $1.07 \times 10^{+9}$ \\
$\widehat{M_{C}}(-10 \mathrm{~V})$ & $6.05 \times 10^{+8}$ & $6.95 \times 10^{+8}$ \\
$\widehat{M_{C}}(-20 \mathrm{~V})$ & $5.40 \times 10^{+8}$ & $6.52 \times 10^{+8}$ \\
$\widehat{M_{C}}(-30 \mathrm{~V})$ & $5.70 \times 10^{+8}$ & $6.84 \times 10^{+8}$ \\
$\widehat{M_{C}}(-40 \mathrm{~V})$ & $5.50 \times 10^{+8}$ & $6.59 \times 10^{+8}$ \\
$\widehat{M_{C}}(-50 \mathrm{~V})$ & $4.90 \times 10^{+8}$ & $5.75 \times 10^{+8}$ \\
\hline
\end{tabular}

\subsubsection{Experiment D: Parameter Extraction}

A parameter extraction procedure is now applied to current-voltage characteristics of a [poly(9,9-dioctylfluoreneco-bithiophene)] (F8T2) based OTFT designed as phototransistor [73]. The structure used by these authors corresponds to an inverted, defined-gate, gate-planarized, coplanar TFT. The source and drain contacts were made of indium tin oxide (ITO), the channel length $L=56 \mu \mathrm{m}$ and the channel width $W=116 \mu m$. Benzocyclobutene (BCB) was used as the gateplanarization layer, also acting as a gate insulator. PECVD hydrogenated amorphous silicon nitride (a-SiN:H) was used as a second gate insulator layer. The effective insulator thickness is $270 \mathrm{~nm}$ and the effective relative dielectric constant 2.3. Chromium (Cr) was used for the patterned gate electrode. The device was fabricated on a silicon substrate. A 1-wt \% solution of F8T2 alternating copolymer dissolved in either xylenes or mesitylenes was used. A 1000 - $\AA$-thick polymer film was spincoated and was cured in a vacuum oven at $90^{\circ} \mathrm{C}$. The output and transfer characteristics measured by these authors in the phototransistors at $2.9 \mathrm{~W} / \mathrm{cm}^{2}$ illumination [73] are represented with crosses in Fig. 9a and 9b, respectively.

The aim of this experiment is not to analyze the effect of illumination but to extend the validity of our method to experimental data in different transistors operating in different conditions. The incorporation of the subthreshold region shown in the transfer characteristics (Figure 9b) adds complexity to the procedure. In order to reproduce the subthreshold region of Figure 9b, Equations (9)-(10) in [45] must be used instead of model (1). These equations are an asymptotically interpolation function of (1), and include a new parameter $V_{S S}$ that controls the subthreshold slope.

The NSGA-II execution parameters defined for this experiment $D$ are shown in Table 1 and the parameters of the bisection method are in Table 2. As in experiment $A$, values of $m_{k}$ from 1.0 to 2.0 with a 0.1 step are tested, but only the $m_{k}$ value that produces the best parameter set is selected. For the remaining parameters, the search space is defined as: 
Table 11: Extracted parameter set for the F8T2 OPTFT ( $k^{\prime}$ is in A/ $\mathrm{V}^{2+\gamma}, V_{T}$ in $\mathrm{V}, V_{S S}$ in $\mathrm{V}$, and $\widehat{M_{C}}\left(V_{G}\right)$ in $\left.\mathrm{A} / \mathrm{V}^{m_{k}}\right)$.

\begin{tabular}{cc}
\hline$x$ & Value \\
\hline$m_{k}$ & $1.00 \times 10^{0}$ \\
$k^{\prime}$ & $2.37 \times 10^{-10}$ \\
$\gamma$ & $8.81 \times 10^{-2}$ \\
$V_{T}$ & $-9.94 \times 10^{0}$ \\
$V_{S S}$ & $5.34 \times 10^{+1}$ \\
$\widehat{M_{C}}(-10 \mathrm{~V})$ & $1.20 \times 10^{-10}$ \\
$\widehat{M_{C}}(-20 \mathrm{~V})$ & $2.44 \times 10^{-10}$ \\
$\widehat{M_{C}}(-30 \mathrm{~V})$ & $5.60 \times 10^{-10}$ \\
$\widehat{M_{C}}(-40 \mathrm{~V})$ & $1.02 \times 10^{-09}$ \\
\hline
\end{tabular}

$$
\begin{array}{r}
k^{\prime} \in\left[10^{-12}, 10^{-08}\right] \mathrm{A} / \mathrm{V}^{(2+\gamma)} ; \gamma \in[0.0,2.0] ; \\
V_{T} \in[-25,15] \mathrm{V} ; V_{S S} \in[0,20] \mathrm{V} ; \\
\widehat{M_{C}}\left(V_{G_{i}}\right) \in\left[10^{-10}, 10^{-04}\right] \mathrm{A} / \mathrm{V}^{m_{k}}
\end{array}
$$

Again, the respective analysis of the obtained Pareto front set is conducted selecting as final result its best solution, or Pareto solution, $f=\left(4.46 \times 10^{-2}, 7.54 \times 10^{-2}\right)$. The solution is represented in Fig. 9 and its estimated parameter set in Table 11. A good agreement between the experimental and estimated $I_{D}-V_{D}$ and $I_{D}-V_{S}$ curves is observed in Fig. 9a and $9 \mathrm{c}$ respectively. The carrier mobility used in the fitting is $\mu_{0}=3.14 \times 10^{-2} \mathrm{~cm}^{2} / \mathrm{Vs}$, one order of magnitude greater than the one obtained if using the ideal MOS model and ignoring the contact effects [73].

The transfer characteristics of Fig. 9b are an additional test of our procedure, showing a very good agreement between experimental (symbols) and estimated (solid line) $I_{D}-V_{G}$ curves, always within the measurement error range. Note that the four points shown with circles in Fig. 9b correspond to values taken from the output characteristics. These points are represented to indicate the possible error ranges in these measurements or other factors not considered in the model, such as dynamic or hysteresis effects. Since the values of the contact parameter $\widehat{M_{C}}$ are extracted for discreet values $V_{G_{i}}$ (symbols in Fig. 9d) a $M_{C}\left(V_{G}\right)$ relation is necessary to calculate the transfer characteristics shown in Fig. 9b. This relation is built by interpolating these discreet values with [11]

$$
M_{C}=\alpha_{2}\left(V_{G}-V_{T}^{\prime}\right)^{(1+\gamma)},
$$

shown in solid line in $9 \mathrm{~d}$, with $\alpha_{2}=1.57 \times 10^{-10}$ and $V_{T}^{\prime}=-8 \mathrm{~V}$, which is consistent with the value of $V_{T}$ in Table 11, and thus, the solution can be considered physically acceptable.

\subsection{Computation costs}

Since the main aim of this work is to prove that our evolutionary procedure can be employed as both, a parameter extraction

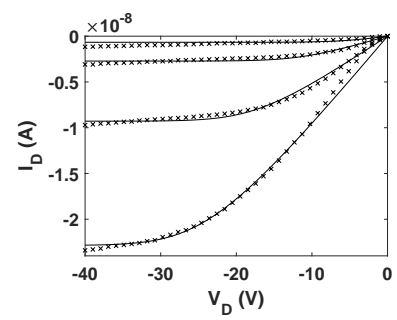

(a)

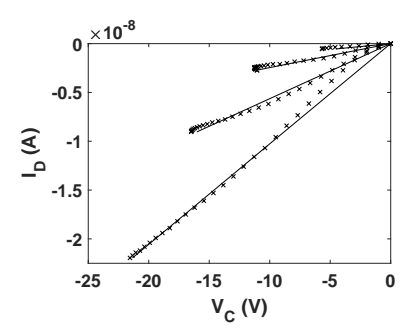

(c)

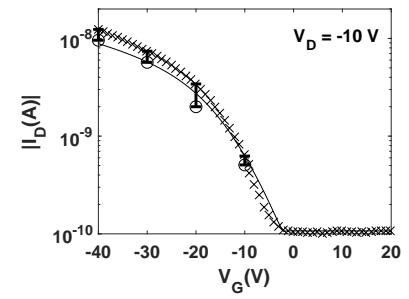

(b)

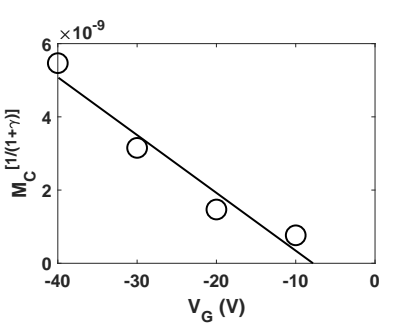

(d)
Figure 9: Experiment D. (a), (b) Comparison of experimental output and transfer characteristics, respectively, (symbols) and the numerical evaluation of (1) (modified to include the subthreshold region [45]) and including the parameter set of Table 11 (solid lines). (c) Current-voltage curves at the contact extracted from (6) and the experimental output characteristics $(\times)$. The solid lines are the fittings using (2) and the estimated parameter set of Table 11. (d) Extracted values of $\widehat{M_{C}}$. The solid line show the trend that the $\widehat{M_{C}}$ set follows according to (26), with $V_{T}^{\prime}=-8 \mathrm{~V}$. In (a) and (c) $V_{G}$ is swept from -10 to $-40 \mathrm{~V}$ with a $-10.0 \mathrm{~V}$ step (from top to bottom). In (b), $V_{D}=-10 \mathrm{~V}$.

method and an optimization procedure, the computation time is not considered a critical point in the EA definition. Even so, our evolutionary procedure reduces the time needed to find a solution with regard to previous extraction procedures. The evolutionary procedure could be used to optimize the solutions presented in this work even further at the cost of increasing the number of iterations, and thus the computational time. Nevertheless, this effort is unnecessary when the error between the calculated and experimental data, is equal or lower than the measurement error, as shown in Fig. 9b.

After the evolutionary procedure execution, it was estimated that for the experiment $A$ each job took $3.5 \pm 1.4$ hours, for the experiment $B 2.4 \pm 0.7$ hours and for experiment $C$ took $28 \pm 3$ minutes for each job. Finally for experiment $D$ each job took $3.3 \pm 1.4$ hours. The difference in the execution time is mainly caused by the different number of points used to evaluate the fitting function.

It is very difficult to quantify the amount of time employed to find an acceptable solution with previous extraction procedures. In the situations in which the $V_{T}$ and $\gamma$ values estimated by the $H_{V G}$ function are invalid, the previous extraction procedures may not find a solution (case exposed in Section 2). In other cases, the search of the remaining parameters by previous extraction procedures could start from an invalid or bad seed, taking too much time to converge or even not converging at all. On the contrary, the convergence of our evolutionary procedure is ensured for the exposed cases, even starting from a bad or invalid seed (initial population randomly generated). 


\section{Conclusion}

We have used an evolutionary parameter extraction procedure to extract device parameters in OTFTs affected by contact effects. This procedure extends the applicability of compact models in TFTs as it provides solutions to some of the limitations of previous parameter extraction procedures.

Once the free parameters of the algorithm have been fixed (number of individuals in each generation, the mutation rate, etc), the process is fixed, and does not depend on a devicespecific expert-based application of elaborated equations and procedures. Also complex expert knowledge must not be provided to start the search, since the evolutionary procedure is capable of converging from a random point (random initial population), being necessary to define an acceptable physical margin for each parameter. Thus, the use of the evolutionary procedure ensures the extraction of reliable and accurate solutions since the evolutionary procedure explores completely its defined search space and will always reach an estimation of an OTFT parameter set.

Also, by exploring the search space defined around a previously known solution, estimated by evolutionary or other heuristic procedures, the proposed evolutionary procedure is capable of optimizing it. The evolutionary procedure has been successfully employed as extraction and optimization method for OTFTs, providing accurate model parameters.

Finally, it is worth mentioning that the solution quality and the execution time of our evolutionary procedure can be improved with parallel implementations of evolutionary algorithms, such as the island-based or the master/worker models. These mechanisms were not considered here as they increase the complexity of the evolutionary procedure, avoiding the reader to keep an easy understanding of it. For these reasons, and since our evolutionary procedure has been successfully applied and its basic ideas have been properly introduced, improvements in the result accuracy and execution time by means of the parallelization of the proposed evolutionary procedure will be considered in future works.

\section{References}

[1] Organic Electronics Association, et al., OE-A roadmap for organic and printed electronics, White Paper.

[2] K. Takimiya, M. Nakano, H. Sugino, I. Osaka, Design and elaboration of organic molecules for high field-effect-mobility semiconductors, Synthetic Metals 217 (2016) 68-78.

[3] T. Uemura, C. Rolin, T.-H. Ke, P. Fesenko, J. Genoe, P. Heremans, J. Takeya, On the extraction of charge carrier mobility in high-mobility organic transistors, Advanced Materials 28 (1) (2016) 151-155.

[4] K. Fukuda, Y. Takeda, M. Mizukami, D. Kumaki, S. Tokito, Fully solution-processed flexible organic thin film transistor arrays with high mobility and exceptional uniformity, Scientific reports 4 (2014) 3947.

[5] R. Picos, E. Garcia, M. Estrada, A. Cerdeira, B. Iñiguez, Effect of process variations on an OTFT compact model parameters, International Journal of High Speed Electronics and Systems 20 (04) (2011) 815-828.

[6] P. V. Necliudov, M. S. Shur, D. J. Gundlach, T. N. Jackson, Modeling of organic thin film transistors of different designs, J. Appl. Phys. 88 (11) (2000) 6594-6597.

[7] D. Gundlach, L. Zhou, J. Nichols, T. Jackson, P. Necliudov, M. Shur, An experimental study of contact effects in organic thin film transistors, J. Appl. Phys. 100 (2) (2006) 024509.
[8] M. J. Deen, M. Kazemeini, S. Holdcroft, Contact effects and extraction of intrinsic parameters in poly(3-alkylthiophene) thin film field-effect transistors, J. Appl. Phys. 103 (12) (2008) 124509.

[9] P. Lara, J. A. Jiménez Tejada, S. Rodríguez-Bolivar, M. J. Deen, O. Marinov, Model for the injection of charge through the contacts of organic transistors, J. Appl. Phys. 105 (8) (2009) 084516.

[10] W. Liu, C. Hu, BSIM4 and MOSFET modeling for IC simulation, World Scientific, 2011

[11] J. A. Jiménez Tejada, J. A. López Villanueva, P. López Varo, K. M. Awawdeh, M. J. Deen, Compact modeling and contact effects in thin film transistors, IEEE Trans. Electron Devices 61 (2) (2014) 266-277.

[12] F. Torricelli, M. Ghittorelli, M. Rapisarda, A. Valletta, L. Mariucci, S. Jacob, R. Coppard, E. Cantatore, Z. Kovács-Vajna, L. Colalongo, Unified drain-current model of complementary p- and n-type OTFTs, Org. Electron. 22 (Supplement C) (2015) 5-11.

[13] A. Valletta, M. Rapisarda, S. Calvi, G. Fortunato, M. Frasca, G. Maira, A. Ciccazzo, L. Mariucci, A DC and small signal AC model for organic thin film transistors including contact effects and non quasi static regime, Org. Electron. 41 (Supplement C) (2017) 345-354.

[14] R. Picos, E. Garcia-Moreno, M. M. Al Chawa, L. O. Chua, Using memristor formalism in semiconductor device modeling, in: Meeting Abstracts, no. 45, The Electrochemical Society, 2017, pp. 2048-2048.

[15] R. Picos, O. Calvo, B. Iñiguez, E. García-Moreno, R. García, M. Estrada, Optimized parameter extraction using fuzzy logic, Solid-state electronics 51 (5) (2007) 683-690.

[16] J. A. Jiménez Tejada, K. M. Awawdeh, J. A. López Villanueva, J. E. Carceller, M. J. Deen, N. B. Chaure, T. Basova, A. K. Ray, Contact effects in compact models of organic thin film transistors: Application to zinc phthalocyanine-based transistors, Org. Electron. 12 (5) (2011) 832-842.

[17] J. A. Jiménez Tejada, P. López Varo, A. N. Cammidge, I. Chambrier, M. J. Cook, N. B. Chaure, A. K. Ray, Compact modeling of organic thin-film transistors with solution processed octadecyl substituted tetrabenzotriazaporphyrin as an active layer, IEEE Trans. Electron Devices 64 (6) (2017) 2629-2634

[18] M. Fayez, K. M. Morsi, M. N. Sabry, OTFTs compact models: analysis, comparison, and insights, IET Circuits, Devices Systems 11 (5) (2017) 409-420.

[19] K. M. Awawdeh, J. A. Jiménez Tejada, P. López Varo, J. A. López Villanueva, F. M. Campos, M. J. Deen, Characterization of organic thin film transistors with hysteresis and contact effects, Org. Electron. 14 (12) (2013) 3286-3296.

[20] K. D. Holland, A. U. Alam, N. Paydavosi, M. Wong, C. M. Rogers, S. Rizwan, D. Kienle, M. Vaidyanathan, Impact of contact resistance on the $f_{t}$ and $f_{\max }$ of graphene versus $\mathrm{MoS}_{2}$ transistors, IEEE Trans. Nanotechnol. 16 (1) (2017) 94-106.

[21] S. V. Suryavanshi, E. Pop, S2DS: Physics-based compact model for circuit simulation of two-dimensional semiconductor devices including nonidealities, J. Appl. Phys. 120 (22) (2016) 224503.

[22] C. Rolin, E. Kang, J. H. Lee, G. Borghs, P. Heremans, J. Genoe, Charge carrier mobility in thin films of organic semiconductors by the gated van der pauw method, Nat. Commun. 8 (2017) 14975.

[23] D. Dasgupta, Z. Michalewicz, Evolutionary Algorithms in Engineering Applications, Springer Publishing Company, Incorporated, 2014.

[24] J. Gero, V. Kazakov, T. Schnier, Genetic Engineering and Design Problems, Springer Berlin Heidelberg, Berlin, Heidelberg, 1997, pp. 47-68.

[25] S. Louis, F. Zhao, X. Zeng, Flaw Detection and Configuration with Genetic Algorithms, Springer Berlin Heidelberg, Berlin, Heidelberg, 1997, pp. 103-116.

[26] D. Dasgupta, Optimal Scheduling of Thermal Power Generation Using Evolutionary Algorithms, Springer Berlin Heidelberg, Berlin, Heidelberg, 1997, pp. 317-328.

[27] G. Antoun, M. El-Nozahi, W. Fikry, H. Abbas, A hybrid genetic algorithm for MOSFET parameter extraction, in: CCECE 2003 - Canadian Conference on Electrical and Computer Engineering. Toward a Caring and Humane Technology (Cat. No.03CH37436), Vol. 2, 2003, pp. 11111114

[28] B. Gross, P. Roosen, Total process optimization in chemical engineering with evolutionary algorithms, Comput. Chem. Eng. 22 (Supplement 1) (1998) S229-S236, european Symposium on Computer Aided Process Engineering-8.

[29] D. Carroll, Chemical laser modeling with genetic algorithms, AIAA J. 
34 (2) (1996) 338-346.

[30] K. Deb, GeneAS: A Robust Optimal Design Technique for Mechanical Component Design, Springer Berlin Heidelberg, Berlin, Heidelberg, 1997, pp. 497-514.

[31] J. Jervase, H. Bourdoucen, A. Al-Lawati, Solar cell parameter extraction using genetic algorithms, Meas. Sci. Technol. 12 (11) (2001) 1922-1925.

[32] J. Alcalá-Fdez, L. Sánchez, S. García, M. J. del Jesús, S. Ventura, J. M. Garrell, J. Otero, C. Romero, J. Bacardit, V. M. Rivas, J. C. Fernández, F. Herrera, Keel: A software tool to assess evolutionary algorithms for data mining problems, Soft Comput. 13 (3) (2008) 307-318.

[33] D. Dracopoulos, Genetic Algorithms and Genetic Programming for Control, Springer Berlin Heidelberg, Berlin, Heidelberg, 1997, pp. 329-343.

[34] B. F. V. Kreinovich, R. Vasquez, Signal Design for Radar Imaging in Radar Astronomy: Genetic Optimization, Springer Berlin Heidelberg, Berlin, Heidelberg, 1997, pp. 409-423.

[35] R. Picos, M. Roca, O. Calvo, E. Garcia-Moreno, Characterisation of radiation effects on MOSFET using genetic algorithms, in: Devices, Circuits and Systems, 2000. Proceedings of the 2000 Third IEEE International Caracas Conference on, IEEE, 2000, pp. D52-1.

[36] J. Watts, C. Bittner, D. Heaberlin, J. Hoffmann, Extraction of compact model parameters for ULSI MOSFETs using a genetic algorithm, in: Proceedings of Second International Conference on Modeling and Simulation of Microsystems, Cambridge, MA, November, 1999, pp. 176-179.

[37] M. Keser, K. Joardar, M. Motorola, Genetic algorithm based MOSFET model parameter extraction, in: Technical Proceedings of the 2000 International Conference on Modeling and Simulation of Microsystems, 2000, pp. 341-344.

[38] Y. Li, Y. Y. Cho, Parallel genetic algorithm for SPICE model parameter extraction, in: Proc. 20th IEEE IPDPS, 2006, p. 8.

[39] M. Siddiqui, M. Abido, Parameter estimation for five- and sevenparameter photovoltaic electrical models using evolutionary algorithms, Appl. Soft Comput. 13 (12) (2013) 4608-4621.

[40] D. Halboot, A. Bakar, T. Khatib, I. Ahmed, Parameters extraction of double diode photovoltaic module's model based on hybrid evolutionary algorithm, Energy Conversion and Management 105 (Supplement C) (2015) 552-561.

[41] N. Moldovan, R. Picos, E. Garcia-Moreno, Parameter extraction of a solar cell compact model usign genetic algorithms, in: Electron Devices, 2009. CDE 2009. Spanish Conference on, IEEE, 2009, pp. 379-382.

[42] P. Moreno, R. Picos, M. Roca, E. Garcia-Moreno, B. Iniguez, M. Estrada, Parameter extraction method using genetic algorithms for an improved OTFT compact model, in: Electron Devices, 2007 Spanish Conference on, IEEE, 2007, pp. 64-67.

[43] R. Picos, E. Garcia-Moreno, B. Iniguez, Non-traditional OTFT parameter extraction, in: Meeting Abstracts, no. 23, The Electrochemical Society, 2007, pp. 1240-1240.

[44] E. Garcia-Moreno, B. Iniguez, R. Picos, Directed genetic algorithms for OTFT model parameter extraction, in: Solid-State and Integrated-Circuit Technology, 2008. ICSICT 2008. 9th International Conference on, IEEE, 2008, pp. 424-427.

[45] O. Marinov, M. Deen, U. Zschieschang, H. Klauk, Organic thin-film transistors: Part I-Compact DC modeling, IEEE Trans. Electron Devices 56 (12) (2009) 2952-2961.

[46] M. Deen, O. Marinov, U. Zschieschang, H.Klauk, Organic thin-film transistors: Part II-Parameter extraction, IEEE Trans. Electron Devices 56 (12) (2009) 2962-2968.

[47] B. H. Hamadani, D. Natelson, Nonlinear charge injection in organic fieldeffect transistors, J. Appl. Phys. 97 (6) (2005) 064508.

[48] P. López Varo, J. A. Jiménez Tejada, J. A. López Villanueva, J. E. Carceller, M. J. Deen, Modeling the transition from ohmic to space charge limited current in organic semiconductors, Org. Electron. 13 (9) (2012) 1700-1709.

[49] A. Cerdeira, M. Estrada, R. García, A. Ortiz-Conde, F. G. Sánchez, New procedure for the extraction of basic a-Si:H TFT model parameters in the linear and saturation regions, Solid-State Electron. 45 (7) (2001) 10771080

[50] R. L. Burden, D. J. Faires, Numerical analysis, 3rd Edition, PWS Publishing Company, Boston, 1985.

[51] A. Ortiz-Conde, F. J. García-Sánchez, J. Muci, A. T. Barrios, J. J. Liou, C.-S. Ho, Revisiting MOSFET threshold voltage extraction methods, Microelectronics Reliability 53 (1) (2013) 90-104.
[52] M. Deen, Z. Yan, A new method for measuring the threshold voltage of small-geometry MOSFETs from subthreshold conduction, Solid-State Electronics 33 (5) (1990) 503 - 511.

[53] Z. Yan, M. Deen, Physically-based method for measuring the threshold voltage of MOSFETs, IEE Proceedings G (Circuits, Devices and Systems) 138 (1991) 351-357(6)

[54] Y. H. Hu, S. Pan, Saposm: an optimization method applied to parameter extraction of MOSFET models, IEEE Transactions on Computer-Aided Design of Integrated Circuits and Systems 12 (10) (1993) 1481-1487.

[55] T. Sakurai, B. Lin, A. R. Newton, Fast simulated diffusion: an optimization algorithm for multiminimum problems and its application to MOSFET model parameter extraction, IEEE Transactions on Computer-Aided Design of Integrated Circuits and Systems 11 (2) (1992) 228-234.

[56] R. Picos, M. Roca, B. Iñiguez, E. Garcia-Moreno, A new MOSFET parameter extraction method using genetic algorithms, in: 1999 Proceedings of CDE, 1999.

[57] R. A. Thakker, N. Gandhi, M. B. Patil, K. G. Anil, Parameter extraction for PSP MOSFET model using particle swarm optimization, in: 2007 International Workshop on Physics of Semiconductor Devices, 2007, pp. 130-133.

[58] X. Yu, M. Gen, Introduction to evolutionary algorithms, Springer, 2010.

[59] A. Eiben, J. Smith, Introduction to evolutionary computing, Vol. 53, Springer, 2003.

[60] C. L. Hwang, A. S. M. Masud, Multiple objective decision making-methods and applications: a state-of-the-art survey, Vol. 164, Springer, 2012.

[61] V. Pareto, Cours d'économie politique, Vol. 1, Librairie Droz, 1964.

[62] K. Deb, A. Pratap, S. Agarwal, T. Meyarivan, A fast and elitist multiobjective genetic algorithm: NSGA-II, Trans. Evol. Comp 6 (2) (2002) 182-197.

[63] E. Zitzler, M. Laumanns, L. Thiele, SPEA2: Improving the strength pareto evolutionary algorithm, Tech. rep., TIK (2001).

[64] C. Fonseca, P. Fleming, Genetic algorithms for multiobjective optimization: Formulationdiscussion and generalization, in: Proc. of the 5th ICGA, Morgan Kaufmann Publishers Inc., San Francisco, CA, USA, 1993, pp. 416-423.

[65] J. Horn, N. Nafpliotis, D. Goldberg, Multiobjective optimization using the niched pareto genetic algorithm, Tech. Rep. 93005, Department of Computer Science, University of Illinois at Urbana-Champaign (Jul. 1993).

[66] D. Goldberg, K. Deb, A comparative analysis of selection schemes used in genetic algorithms, in: Foundations of Genetic Algorithms, Morgan Kaufmann, 1991, pp. 69-93.

[67] R. B. Agrawal, K. Deb, Simulated binary crossover for continuous search space, Complex Systems 9 (2) (1995) 115-148.

[68] H.-G. Beyer, K. Deb, On self-adaptive features in real-parameter evolutionary algorithms, Trans. Evol. Comp 5 (3) (2001) 250-270.

[69] K. Deb, S. Agrawal, A Niched-Penalty Approach for Constraint Handling in Genetic Algorithms, Springer Vienna, Vienna, 1999, pp. 235-243.

[70] K. Deb, D. Deb, Analysing mutation schemes for real-parameter genetic algorithms, Int. J. Artif. Intell. Soft Comput. 4 (1) (2014) 1-28.

[71] J. González, I. Rojas, J. Ortega, H. Pomares, F. Fernandez, A. Díaz, Multiobjective evolutionary optimization of the size, shape, and position parameters of radial basis function networks for function approximation, IEEE Trans. Neural Netw. 14 (6) (2003) 1478-1495.

[72] S. Luke, ECJ then and Now, in: Proc. of the GECCO, GECCO '17, ACM, New York, NY, USA, 2017, pp. 1223-1230.

[73] M. C. Hamilton, S. Martin, J. Kanicki, Thin-film organic polymer phototransistors, IEEE Transactions on Electron Devices 51 (6) (2004) 877885 . 